\title{
ON A RECENT METHOD FOR DEALING WITH THE INTERSECTIONS
}

\section{OF PLANE CURVES*}

\author{
BY \\ CHARLOTTE ANGAS SCOTT
}

\section{Introduction.}

1. In considering NoETheR's theorem under its geometrical aspect, a question of interpretation forces itself into notice. In order that a curve $F=0$ may have an equation of the form $P u+Q v=0$, where $u=0, v=0$ are given curves, the coefficients in the expression $F$ must satisfy certain conditions ; any one intersection of $u, v$ being taken as origin, the conditions arising from this one affect the coefficients of terms whose degree does not exceed a certain value. Of these conditions some bear their interpretation on their face-the curve $F$ must have a multiple point of a certain order, with tangents possibly given; but what is the geometric meaning of the others?

The determination of their precise algebraic construction is the first stage in the inquiry, and to this question an answer has been given in a very simple and significant form in a recent memoir by Dr. F. S. MACAUlay. $\dagger$ His explanation of the nature of the conditions is applicable not only to the so-called simple case, when the two curves $u, v$ have no contact at any common point, but also to the general case, when either curve alone presents a singularity of any degree of complexity, and the two have contact of however elaborate a nature.

The conditions are simply the vanishing of $(1)$ a single linear function of the coefficients, and (2) all functions obtained from it by a particular process of derivation. If we denote the coefficient of $x^{r} y^{q}$ by $z_{r, q}$, or, more conveniently, the coefficient of $x^{p-q} y^{q}$ by $z_{q}^{p}$, this process is simply the repeated and combined use of the two operators:

$D_{x} \equiv$ diminish every $r$ by unity, $D_{y} \equiv$ diminish every $q$ by unity, or,

$D_{x} \equiv$ diminish every index $p$ by unity, with the understanding that $z_{q}^{p}$ is zero, for $p<q$,

$D_{\nu} \equiv$ diminish every index $p$ and suffix $q$ by unity.

* Presented to the Society December 27, 1901. Received for publication February 11, 1902.

†Proceedings of the London Mathematical Society, vol. 31 (1899), pp. 381423 ; see also vol. 32 (1900), pp. 418-430. 
The order in which the operators are used is obviously a matter of absolute indifference. Thus, for example, if the original condition is $E=0$, where

then

$$
E=a z_{0}^{3}+b z_{1}^{3}+c z_{2}^{3}+d z_{3}^{3}+e z_{0}^{2}+f z_{1}^{2}+g z_{2}^{2}+h z_{0}^{1}+k z_{1}^{1}+l z_{0}^{0},
$$

$$
\begin{aligned}
D_{x} E & =a z_{0}^{2}+b z_{1}^{2}+c z_{2}^{2}+e z_{0}^{1}+f z_{1}^{1}+h z_{0}^{0}=0, \\
D_{y} E & =b z_{0}^{2}+c z_{1}^{2}+d z_{2}^{2}+f z_{0}^{1}+g z_{1}^{1}+k z_{0}^{0}=0, \\
D_{x}^{2} E & =a z_{0}^{1}+b z_{1}^{1}+e z_{0}^{0}=0, \\
D_{x} D_{y} E & =b z_{0}^{1}+c z_{1}^{1}+f z_{0}^{0}=0, \\
D_{y}^{2} E & =c z_{0}^{1}+d z_{1}^{1}+g z_{0}^{0}=0,
\end{aligned}
$$

$D_{x}^{3} E=a z_{0}^{0}=0, \quad D_{x}^{2} D_{y} E=b z_{0}^{0}=0, \quad D_{x} D_{y}^{2} E=c z_{0}^{0}=0, \quad D_{y}^{3} E=d z_{0}^{0}=0$.

These four equations reduce to the one, $z_{0}^{0}=0$; the three above are equivalent to two only, namely, $z_{0}^{1}=0, z_{1}^{1}=0$, unless such relations hold among $a, b, c, d$ as will reduce these three to one, namely, $a / b=b / c=c / d$. The two before these give

that is

$$
a z_{0}^{2}+b z_{1}^{2}+c z_{2}^{2}=0, \quad b z_{0}^{2}+c z_{1}^{2}+d z_{2}^{2}=0,
$$

$$
z_{0}^{2}: z_{1}^{2}: z_{2}^{2}=b d-c^{2}: b c-a d: a c-b^{2}
$$

and the original equation can be simplified by the omission of the terms now known to be zero. Thus it is seen that there is a double point with given tangents, the coefficients of the tern, of the third degree being moreover subject to one linear condition. The number of independent equations in this system is six.

The set of equations, consisting of a single "prime equa ion" and all its derivates, Dr. Macaulay speaks of as a "one-set system"; the "base-point" thus defined (that is, the point together with the entire specified nature of the curve) he calls a "one-set point." If $t$ prime equations are necessary for the complete specification, the point is a " $t$-set point."

2. The second of the two memoirs contains certain applications of the theory which is developed in the first memoir. The principal theorems there obtained, but in a different order, are :

(1) the complete intersection of two curves $u, v$ determines a one-set point (pp. $394-400)$;

(2) the complete intersection of $t+1$ curves $u_{0}, u_{1}, \cdots, u_{t}$ determines a $t$-set point (pp. 419-423);

(3) the number of points of intersection of two curves at one common point is the same as the number of equations contained in the one-set system afforded by the intersection (pp. 388-393). 
The whole development of the theory, as well as the proofs of these theorems, is elaborate and complicated ; for instance, certain theorems of residuation are proved, and on these the proof of (2) is based. These theorems are, however, far removed from the inherent simplicity of the conception, and they throw.no light on the very interesting character of the equations. On account of the intrinsic interest, and, I believe, importance of the central idea, it seems worth while attempting to present the theory with some fulness, but in a more direct manner. In this recasting of Dr. MACAULAY's material I have slightly inverted the definitions of his original memoir, and have given entirely different proofs of theorems (1) and (2). These proofs are in a different order of ideas; by means of a theorem of ascent $\mathrm{I}$ determine with precision the nature of the set of equations, after which examination the proofs of the two theorems follow immediately. The proof of (3) (Theorem I in the original memoir), is practically the same as the proof there given, but the preliminary investigation into the nature of the equations makes it somewhat more simple.*

3. Some preliminary remarks will perhaps make the trend of the argument clearer. As these are simply of a general and explanatory character, by no means essential to the formal treatment, some freedom is exercised in the use of certain phrases, which in a different context might challenge criticism. We begin by postulating elements of which the simplest kind is the usual directed linear infinitesimal element; these are combinations of infinitesimal arcs of all possible kinds, connected (as members of one complete branch) or entirely disconnected. Thus, for example, two ares through the origin determine a double point; if no information is given as to the tangents, this element has two initial degrees of freedon ; if the tangents are to be a pair in an assigned involution of line-pairs, the element has only one initial degree of freedom. We are concerned only with the degrees of freedom of the element assigned by the given conditions; we have nothing to do with what happens to the separate arcs afterwards. Such an element is spoken of as a base-point. If the element has one initia degree of freedom, the base-point is a one-set point; if it has $t$ degrees of freedom, the oint is $t$-set. Thus an unconditioned $k$-point (multiple point of order $k$ ) is necessarily a $k$-set point, for the element is composed of $k$ simple ares with unspecified tangents, and has, therefore, $k$ degrees of freedom. But if any relation is to hold among the branches, this diminishes the number of degrees of freedom, and the point is a $t$-set point, where $t<k$. Any branch may be separately specified to any extent; but if then left free to wave independently of the others, the result still holds.

* The proof as given in the memoir, under its most natural interpretation (for it is extremely obscure) is open to oriticism also on the soore of rigor, but $I$ have received from the author a modified form of some of the statements, designed to make it clear that when properly interpreted they are not open to this criticism. The obscurity however remains. 
If the point be $t$-set, let $u_{0}, u_{1}, \cdots, u_{t}$ be curves, otherwise independent, on which it exists; then it exists also on

$$
X_{0} u_{0}+X_{1} u_{1}+\cdots+X_{t} u_{t}=0,
$$

where $X_{0}, X_{1}, \cdots, X_{t}$ are general polynomials in $x, y$; and as this has initially $t$ degrees of freedom at the origin the system may be expected to include all curves endowed with this point.

The equations proper to any base-point may be looked upon as specifying the nature of the curve in an infinitesimal region surrounding the point; when the curve enters this region, it comes under the influence of the prime equations, $t$ in number if the point is $t$-set; but it is by no means necessary that the curve come under the influence of all $t$ prime equations simultaneously.

\section{Nature and arrangement of the equations.}

4. Our object is to determine the nature of the conditions to which the coefficients in the equation of a curve, $u=0$, must be subject in order that the equation may be expressible in the form $X_{0} u_{0}+X_{1} u_{1}=0$, or, we may say, in order that the curve may be a member of the system $X_{0} u_{0}+X_{1} u_{1}=0$, where $u_{0}, u_{1}$ are given curves, while $X_{0}, X_{1}$ are entirely arbitrary polynomials in $x, y$. We consider this question only as regards the conditions due to the nature of the curves at the origin, this having been taken at a point common to $u_{0}, u_{1}$. All curves of the system have something in common at the origin, even if it be only, as in the simplest case, that they pass through it. Similarly, as regards the more general system of the same type,

$$
X_{0} u_{0}+X_{1} u_{1}+X_{2} u_{2}+\cdots=0
$$

which obviously includes the general linear system

$$
h_{0} u_{0}+h_{1} u_{1}+h_{2} u_{2}+\cdots=0,
$$

there arises the question of the behavior of the curves at the origin; in other words, the determination of their common characteristics at that point.

The form of the conditions appear at once from this delimitation of the field of investigation. (1) If $X$ be any polynomial, the reducible curve $X u=0$ has the same branches at the origin as $u=0$, with others in addition if the curve $X=0$ itself passes through this point. Hence the coefficients in $X u$ satisfy the equations to which the coefficients of $u$ are subject. Since $X$ may be taken to be a mere numerical multiplier, this proves that every equation is homogeneous in the coefficients of $u$. (2) Inasmuch as any curve $u+k v=0$ belongs to the system if $u, v$ are members, it follows that the coefficients in $u+k v$ satisfy the equations for all values of $k$. Consequently the coefficients of $u$ enter only in the first degree; that is, every equation with which we are concerned is linear 
in "the coefficients of $u$. (3) If for the polynomial $X$ we take simply $x^{h} y^{k}$, it is evident that any equation satisfied by the coefficients of $u$ in virtue of the base-point. is satisfied also by the corresponding coefficients of $x^{h} y^{k} u$.* $^{*}$ Thus if $\sum \lambda_{r, q} z_{r, q}=0$, and if we form this same expression for $x^{h} y^{k} u$, the term $x^{r} y^{q}$ has now the coefficient $z_{r-h, q-k}$, hence we have $\sum \lambda_{r, q} z_{r-h, q-k}=0$. The original equation being denoted by $E=0$, the values 1,0 for $h, k$ give $D_{x} E=0$, and the values 0,1 give $D_{y} E=0$, where $D_{x}, D_{y}$ have the meanings explained in the introduction. The equation derived from $E=0$, as above, is $D_{x}^{h} D_{y}^{k} E=0$. In the index and suffix notation, the result obtained is that if $\sum \lambda_{q}^{p} z_{q}^{p}=0$, then for any set of values $l, m$, such that $p-l \geqq q-m, l \geqq m$, $\sum \lambda_{q}^{p} z_{q-m}^{p-l}=0$. If this be denoted by $E_{m}^{l}$, then $D_{x} E$ is $E_{0}^{1}, D_{y} E$ is $E_{1}^{1}$. The equation $E=0$ is looked upon as the prime equation or source; the others are derivates or descendants. By the degree of an equation is to be understood the highest index $p$ that occurs. If an equation is of degree $p$, then any $p$ th derivate is simply $z_{0}^{0}=0$.

5. One prime equation with all its derivates may not account for all the equations to which the coefficients are subject. If $t$ prime equations are necessary, the point is said to be t-set. For instance, the two prime equations $z_{0}^{1}=0, z_{1}^{1}=0$, with the one derivate $z_{0}^{0}=0$, determine a two-set point, a simple node. If all the equations are accounted for by the one prime equation and its derivates, the point is one-set.

An equation that is prime for one base-point may present itself as a derivate for a more extensive base-point. For example, on the curve

the curve

$$
x+y+x^{2}+3 x y+4 y^{2}+x^{3}+2 x^{4}+y^{4}+\cdots=0,
$$

$$
x+y+2 x^{2}+4 x^{3}+y^{3}+6 x^{4}+\cdots=0
$$

determines a one-set point for which the prime equation is $z_{0}^{2}-z_{1}^{2}+z_{2}^{2}-2 z_{0}^{1}=0$; while the curve

$$
x+y+x^{2}+y^{2}-7 y^{3}+5 x^{4}+2 y^{4}+\cdots=0
$$

determines a one-set point with the prime equation

$$
z_{0}^{3}-z_{1}^{3}+z_{2}^{3}-z_{3}^{3}-2 z_{0}^{2}+2 z_{2}^{2}-7 z_{0}^{1}=0,
$$

whose $x$-derivate is $z_{0}^{2}-z_{1}^{2}+z_{2}^{2}-2 z_{0}^{1}=0$.

* Although this brings in other brauches at $O$, the existing branches are not affected. The wording in the text can, however, be varied ; $\left(1+b x^{k} y^{k}\right) u$ has exactly the same base-point as $u$; hence, if $\Sigma \lambda_{r, q} z_{r, q}=0$, it follows that $\Sigma \lambda_{r, q}\left(z_{r, q}+b z_{r-h, q-k}\right)=0$, from which, by subtraction, the result follows as in the text. 
6. Law of unit increase.-From the mode of formation of the derivates, it is seen that the number of any degree may increase by unity for every diminution in the degree; we have, in fact, the scheme

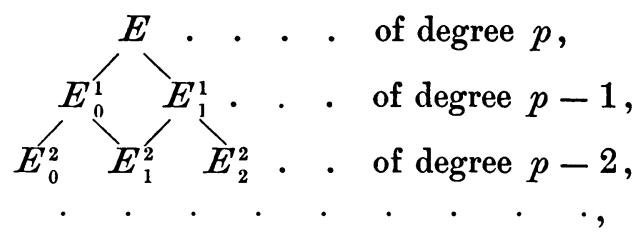

where the two derivates of any equation are placed obliquely below it, the $x$ derivate to the left, the $y$-derivate to the right. Thus $E_{1}^{2}$ is the $x$-derivate of $E_{1}^{1}$ and the $y$-derivate of $E_{0}^{1}$. There may however be agreement among the derivates; in the example of the last paragraph, the $y$-derivate of

$$
z_{0}^{3}-z_{1}^{3}+z_{2}^{3}-z_{3}^{3}-2 z_{0}^{2}+2 z_{2}^{2}-7 z_{0}^{1}=0
$$

is $-z_{0}^{2}+z_{1}^{2}-z_{2}^{2}+2 z_{1}^{1}=0$, which is the same as the $x$-derivate, in virtue of the relation, shown by the next derivates, $z_{0}^{1}-z_{1}^{1}=0$. On account of this possibility, all that can be asserted at present is that in any one-set system the increase, for unit decrease in degree, cannot be more than unity. This warrants no conclusions as to the total number of equations of any degree for a $t$-set point, inasmuch as there may be prime equations of that degree.

7. Whether the point be one-set or t-set, if the greatest number of equations of any one degree be $k$ (where obviously $k \geqq t$ ), the point is multiple of order $k$. By hypothesis, there are $k$ different equations of some degree $p$; that is, from these there can be formed linearly no equation of lower degree. It is to be proved that the number of independent equations of any lower degree $p^{\prime}$ cannot be less than $k$, provided that $p^{\prime} \geqq k$. As every equation gives at least one representative in the next lower degree, it has to be shown that these can be chosen so that no two agree as to their highest terms; or, more generally, so that the highest terms cannot be eliminated from any number of the equations. The choice to be made is that between $x$-derivates and $y$-derivates.

If some of the $x$-derivates can be combined in such a manner as to eliminate the highest terms, then their sources can be similarly combined so as to eliminate the highest terms with the exception of $z_{p}^{p}$; for any term $\lambda_{q}^{p} z_{q}^{p-1}$ in a derivate arises from a term $\lambda_{q}^{p} z_{q}^{p}$ in the source, hence all terms in the source, except $z_{p}^{p}$, are represented in the derivates. This combination of the sources yields an equation by which $z_{p}^{p}$ is given in terms of $z$ 's with a lower index. There cannot be two such equations, since by hypothesis it is not possible to eliminate every $z^{p}$.

Hence in forming the $x$-derivates one of two things must happen : either (1) the $k$ independent equations of degree $p$ give, by means of their $x$-derivates, $k$ inde- 
pendent equations of degree $p-1$, which is the desired result; or (2) the $k$ equations of degree $p$ can be written as one, giving $z_{p}^{p}$ in terms of lower $z$ 's, and $k-1$ that do not contain $z_{p}^{p}$. The $x$-derivates of these give $k-1$ equations, and the $y$-derivate of the first gives $z_{p-1}^{p-1}$ in terms of lower $z$ 's. If this is independent of the $k-1$, the result follows; if it is not, these $k-1$ equations can be combined so as to eliminate every $z^{p-1}$ except $z_{p-1}^{p-1}$, and the similar combination of their sources will not contain any $z^{p}$ except $z_{p-1}^{p}$; this combination is therefore an equation giving $z_{p-1}^{p}$ in terms of lower $z$ 's. The $k$ equations of degree $p$ are now

(1) one, giving $z_{p}^{p}$ in terms of lower $z$ 's,

(2) one, giving $z_{p-1}^{p}$ in terms of lower $z$ 's,

(3) $k-2$, not containing $z_{p}^{p}, z_{p-1}^{p}$.

At every repetition of this argument, either the immediately favorable case presents itself, or we continue with the apparently unfavorable case; this goes on until the $k$ equations of degree $p$ are all arranged so as to give, separately, $z_{p}^{p}, z_{p-1}^{p}, z_{p-2}^{p}, \cdots, z_{p-k+1}^{p}$ in terms of lower $z^{\prime}$ s. The $y$-derivates of these, which are all different, yield $k$ independent equations of degree $p-1$.

This argument holds so long as $p-k+1>0$, that is, down to and including the case $p^{\prime}=k$. Thus we have $k$ equations of the type

$$
\left(z_{0}^{k}, z_{1}^{k}, \cdots z_{i}^{k}\right)^{1}=\text { a linear function of lower } z \text { 's. }
$$

(i) If every $z^{k}$ is present, these $k$ equations can be solved so as to give every $z_{q}^{k}(q \neq k)$ in terms of $z_{k}^{k}$ and lower $z$ 's. The $x$-derivates then give $k$ independent equations

$$
\begin{aligned}
& z_{0}^{k-1}=\text { a linear function of lower } z^{\prime} \mathrm{s}, \\
& z_{1}^{k-1}=\text { a linear function of lower } z^{\prime} \mathrm{s}, \\
& \vdots \\
& z_{k-1}^{k-1}=\text { a linear function of lower } z \text { 's. }
\end{aligned}
$$

Proceeding with the $x$-derivates, we obtain finally, in reverse order,

$$
\begin{aligned}
& z_{0}^{0}=0, \\
& z_{0}^{1}=\left[a z_{0}^{0}=\right] 0, \quad z_{1}^{1}=0, \\
& z_{0}^{2}=\left(z_{0}^{1}, z_{1}^{1}, z_{0}^{0}\right)^{1}=0, z_{1}^{2}=0, \quad z_{2}^{2}=0, \\
& \cdot \quad \cdot \quad \cdot \quad \cdot \quad \cdot \quad \cdot \quad \cdot \quad \cdot \quad \cdot \\
& z_{0}^{k-1}=0, \quad z_{1}^{k-1}=0, \quad z_{2}^{k-1}=0, \quad \cdots, \quad z_{k-1}^{k-1}=0 .
\end{aligned}
$$


These values for the lower $z$ 's reduce the $k$ equations of degree $k$ to the form

$$
z_{0}^{k}: z_{1}^{k}: z_{2}^{k}: \cdots: z_{k}^{k}=\text { known values. }
$$

Thus the point is multiple, of order $k$, with determinate tangents.

(ii) If any one $z^{k}$ be absent, the remaining $z^{k}, \mathrm{~s}, k$ in number, are obtainable linearly in terms of lower $z$ 's. The $x$-derivates of those before the missing one, and the $y$-derivates of those after, give the $k$ independent equations exactly as in the preceding case. We still have the multiple point of order $k$; and moreover every $z^{k}$, except the missing one, is zero. If this unmentioned one be $z_{l}^{k}$, the tangents are $x^{k-l} y^{l}=0$. If now $p<k$, this amounts to saying that we have $k$ independent equations of degree $\leqq k-1$. As the number of $z$ 's of any degree $h$ is equal to $h+1$, the lowest possible value for this degree is $k-1$; the equations in this case can be written so as to give $z_{0}^{k-1}, z_{1}^{k-1}, z_{2}^{k-1}, \ldots, z_{k-1}^{k-1}$ linearly in terms of lower $z$ 's, and exactly as before, by means of $x$-derivates it is seen that every $z$ up to and including every $z^{k-1}$ must vanish. In this case however the tangents are not necessarily determined.

The general conclusion is therefore that if for any degree $p$ there are as many as $k$ independent equations, this being the greatest number for any degree, then the point is multiple of order $k$, with tangents which are determinate, if $p \geqq k$; possibly conditioned in some manner, if $p=k-1$; entirely unconditioned, if $p=k-1$ and the equations are prime.

8. Any equation of the set may of course be modified by the addition of mul. tiples of any other of the equations. When it is found that the point is multiple of order $k$, so that every $z^{p}(p<k)$ is zero, all these lower $z$ 's are to be struck out of the equations. Another possible simplification can sometimes be detected. If a linear function of an expression $E$ and some of its derivates presents itself as an equation of the set, this can be replaced by $E=0$. For taking all the derivates down to and including the $p$ th derivates, where $p$ is the degree of $E$, we obtain

$$
E_{0}^{p}=0, E_{1}^{p}=0, \cdots, E_{p}^{p}=0 ; E_{0}^{p-1}=\text { linear functions of } E^{p} \mathrm{~s}=0,
$$

and so on, till finally $E=0$.

9. The argument by which it was shown that the point is multiple of order $k$ depends on proving that the number of equations cannot diminish as the degree diminishes, so long as this degree $\geqq k$. It has been remarked that in general the number of equations derivable from any one prime equation increases by unity when the degree is diminished by unity. The prime equation $E=0$, of degree $p$, yields two derivates $E_{0}^{1}$ and $E_{1}^{1}$, and consequently two equations of degree $p-1$; thus there is at least a double point, unless these two derivates are absolutely equivalent. To exhibit them as equivalent, it may be necessary 
to modify them by the addition of multiples of lower derivates, but this will not affect the terms of highest degree. Let the prime equation be

$$
E=a_{0} z_{0}^{p}+a_{1} z_{1}^{p}+\cdots+a_{p} z_{p}^{p}+(z)^{p-1}+\cdots=0
$$

the derived equations are

$$
\begin{aligned}
& E_{0}^{1}=a_{0} z_{0}^{p-1}+a_{1} z_{1}^{p-1}+\cdots+a_{p-1} z_{p-1}^{p-1}+(z)^{p-2}+\cdots=0 \\
& E_{1}^{1}=a_{1} z_{0}^{p-1}+a_{2} z_{1}^{p-1}+\cdots+a_{p} z_{p-1}^{p-1}+(z)^{p-2}+\cdots=0 .
\end{aligned}
$$

These agree as to their highest terms if, and only if, $a_{0} / a_{1}=a_{1} / a_{2}=\cdots=a_{p-1} / a_{p}$; that is, if the coefficients of the highest terms form a geometric progression. The prime equation can then be written

$$
z_{0}^{p}+\mu z_{1}^{p}+\mu^{2} z_{2}^{p}+\cdots+\mu^{p} z_{p}^{p}+(z)^{p-1}+\cdots=0,
$$

so that it is at once obvious that all derivates of any one degree are equivalent as regards their highest terms.

The equation $\mu E_{0}^{1}-E_{1}^{1}=0$ is of degree lower than $p-1$; if it is expressible in terms of derivates of $E_{0}^{1}$, the two equations $E_{0}^{1}$ and $E_{1}^{1}$ are absolutely equivalent; from $E=0$ we obtain by a single derivation only the one equation $E_{0}^{1}=0$. Similarly the three derivates of degree $p-2$ reduce to one only; for they are $D_{x} E_{0}^{1}, D_{x} E_{1}^{1}\left(=D_{y} E_{0}^{1}\right), D_{y} E_{1}^{1}$. Now $E_{1}^{1} \equiv E_{0}^{1}$, therefore $D_{y} E_{1}^{1} \equiv D_{y} E_{0}^{1} \equiv D_{x} E_{1}^{1} \equiv D_{x} E_{0}^{1}$; also $D_{x} E_{1}^{1} \equiv D_{x} E_{0}^{1}$, thus all are equivalent to $E_{0}^{2}$. In like manner the next derivates reduce to one only, and so on. Hence unless the first two derivates are independent, there is but one derivate of any degree, and the point is not multiple. If the point determined by a given prime equation is multiple, this fact will make itself felt at the first derivation.

If now the equation $\mu E_{0}^{1}-E_{1}^{1}=0$, of degree $<p-1$, is not expressible in terms of lower derivates of $E_{0}^{1}$, the two equations $E_{0}^{1}$ and $E_{1}^{1}$ are not absolutely equivalent; from $E=0$ we obtain by a single derivation the equation $E_{0}^{1}=0$ and an equation of lower degree. Similarly at any stage in the derivation it may be possible to eliminate from the $m+1$ equations of degree $p^{\prime}-1$ all the highest terms, thus obtaining an equation of degree $p^{\prime \prime}\left(<p^{\prime}-1\right)$, to be substituted for one of the $m+1$ equations. If this new equation is expressible in terms of the derivates of the $m$ others, it adds nothing to our knowledge; from the $m$ equations of degree $p^{\prime}$ we obtain only $m$ equations of degree $p^{\prime}-1$. But if this new equation is not so expressible, it has to be taken into account when we arrive at degree $p^{\prime \prime}$.

10. In forming the scheme of equations, whether it be regular or interrupted, the identity of $D_{x} E_{k}^{l}$ and $D_{y} E_{k-1}^{l}$ makes it unnecessary to write down both $x$ - and $y$-derivates of all the equations at any stage; it is enough to write down 
the $x$-derivates of all, and the $y$-derivates of the last one (the pure $y$-derivate $E_{l}^{l}$ ). The simplest process is perhaps to form the $y$-derivates, writing these in an oblique line downwards to the right, and then write down a vertical line of $x$-derivates, starting from each of these. The simple or regular scheme, that in which no elimination of the highest terms from the equations of any one degree is possible, is then

$$
\begin{aligned}
& E \text {. . . . . of degree } p \text {, } \\
& E_{0}^{1} E_{1}^{1} \quad \cdot \quad \cdot \quad \text {. of degree } p-1 \text {, } \\
& E_{0}^{2} \quad E_{1}^{2} \quad E_{2}^{2} \text {. . . of degree } p-2 \text {, } \\
& \begin{array}{llll}
E_{0}^{3} & E_{1}^{3} & E_{2}^{3} & E_{3}^{3}
\end{array} \text {. of degree } p-3, \\
& \text {. . etc. . . . etc. . . }
\end{aligned}
$$

If now the arrangement is interrupted by the possibility of eliminating the terms of degree $p^{\prime}-1$ from the $m+1$ equations of degree $p^{\prime}-1$, so obtaining an equation $E^{\prime}=0$ of degree $p^{\prime \prime}\left(<p^{\prime}-1\right)$, for the derivates at this stage we can substitute $E_{0}, E_{1}, E_{2}, \ldots, E_{m-1}, E_{m}^{\prime}$. As before, it suffices to take the $x$-derivates of $E_{0}, E_{1}, \cdots, E_{m-1}$, if both $x$ - and $y$-derivates be taken of $E_{m}^{\prime}$. Thus for degrees $p^{\prime}-1$ to $p^{\prime \prime}+1$ included, there are $m$ equations; at degree $p^{\prime \prime}$ there are $m+1$ equations, since $E_{m}^{\prime}$ is now to be taken into account, and the law of unit increase is resumed until again interrupted. It is convenient to speak of the equations of which $x$-derivates only need be taken as stationary, the other being progressive. As an illustration of such an interrupted scheme take the prime equation

$$
\begin{aligned}
E=z_{0}^{8}-z_{1}^{8}- & z_{3}^{8}-z_{4}^{8}-2 z_{5}^{8}-3 z_{6}^{8}-5 z_{7}^{8}-8 z_{8}^{8}+2 z_{0}^{7}+z_{1}^{7}+z_{2}^{7}+3 z_{3}^{7} \\
& +z_{4}^{7}-z_{5}^{7}+z_{0}^{6}+3 z_{1}^{6}+z_{2}^{6}+4 z_{3}^{6}+z_{4}^{6}+3 z_{0}^{5}+2 z_{1}^{5}+z_{2}^{5}+8 z_{0}^{4}=0 .
\end{aligned}
$$

Here $E_{0}^{2}+E_{1}^{2}-E_{2}^{2}$ is of degree 5 ; there are only two independent equations of degree 6 . The three of degree 5 are

and

$$
\begin{aligned}
& E_{0}^{3}=z_{0}^{5}-z_{1}^{5}-z_{3}^{5}-z_{4}^{5}-2 z_{5}^{5}+2 z_{0}^{4}+z_{1}^{4}+z_{2}^{4}+3 z_{3}^{4}+z_{4}^{4}=0, \\
& E_{1}^{3}=-z_{0}^{5}-z_{2}^{5}-z_{3}^{5}-2 z_{4}^{5}-3 z_{5}^{5}+z_{0}^{4}+z_{1}^{4}+3 z_{2}^{4}+z_{3}^{4}-z_{4}^{4}=0,
\end{aligned}
$$

$E_{0}^{2}+E_{1}^{2}-E_{2}^{2}=\ddot{E}=2 z_{0}^{5}-z_{1}^{5}+3 z_{2}^{5}+5 z_{3}^{5}-z_{5}^{5}+3 z_{0}^{4}+4 z_{2}^{4}+5 z_{3}^{4}+z_{4}^{4}=0 ;$

and since there are four of degree 4 , namely

$$
\begin{aligned}
E_{0}^{4} & =z_{0}^{4}-z_{1}^{4}-z_{3}^{4}-z_{4}^{4}=0, \\
E_{1}^{4} & =-z_{0}^{4}-z_{1}^{4}-z_{2}^{4}-2 z_{4}^{4}=0, \\
D_{x} \bar{E} & =2 z_{0}^{4}-z_{1}^{4}+3 z_{2}^{4}+5 z_{3}^{4}=0, \\
D_{y} Z & =-z_{0}^{4}+3 z_{1}^{4}+5 z_{2}^{4}-z_{4}^{4}=0,
\end{aligned}
$$


there is a 4-point, with determinate tangents, given by

that is,

$$
z_{0}^{4}: z_{1}^{4}: z_{2}^{4}: z_{3}^{4}: z_{4}^{4}=82: 94:-45: 13:-25 \text {, }
$$

$$
82 x^{4}+94 x^{3} y-45 x^{2} y^{2}+13 x y^{3}-25 y^{4}=0 .
$$

The diagrammatic representation of this set of equations is

$$
\begin{array}{llllllll}
E & \cdot & \cdot & \cdot & \cdot & \text { of degree } 8, \\
E_{0}^{1} & E_{1}^{1} & \cdot & \cdot & \cdot & \cdot & \text { of degree } 7, \\
E_{0}^{2} & E_{1}^{2} & \cdot & \cdot & \cdot & \cdot & \text { of degree } 6, \\
E_{0}^{3} & E_{1}^{3} & \bar{E} & \cdot & \cdot & \cdot & \text { of degree } 5, \\
E_{0}^{4} & E_{1}^{4} & \bar{E}_{0} & \bar{E}_{1} & \cdot & \text { of degree } 4 .
\end{array}
$$

In the regular scheme, if the prime equation be of degree $p$, the number of equations of any degree $k-1$ is $p+2-k$. So long as $k \geqq p+1-k$, the derivation can go on; but since a set of $k$ equations of degree $k-1$ indicates a $k$-point, the value of $p$ is determined by the equality $k=p+2-k$, and consequently $p=2(k-1)$. If $p$ has a greater value than this, the scheme is not regular.

11. It has been shown that if the first two derivates are absolutely equivalent, then there is only one derivate of any particular degree. The general theorem, of which this is a special case, is the following:

If the $k$ derivates of degree $p$ give rise to only $k$ derivates, then the number remains stationary, and the point is consequently a k-point.

Let the $k$ derivates of degree $p$ be denoted by $E_{0}, E_{1}, E_{2}, \ldots, E_{k-1}$. If the set were regular, we should have at the next stage $k+1$ derivates, $E_{0}^{1}, E_{1}^{1}, E_{2}^{1}, E_{k-1}^{1}, E_{k}^{1}$, where $E_{0}^{1}=D_{x} E_{0}$, etc., and $E_{k}^{1}=D_{y} E_{k-1}$; but by hypothesis, these are equivalent to $k$ only. There is therefore one linear relation connecting some or all of the $E^{1}$ 's; let the last $E^{1}$ involved in this be $E_{h}^{1}$, so that the relation can be written

$$
E_{h}^{1}=a_{0} E_{0}^{1}+a_{1} E_{1}^{1}+a_{2} E_{2}^{1}+\cdots+a_{h-1} E_{h-1}^{1} .
$$

The independent derivates of this rank are now

$$
E_{0}^{1}, E_{1}^{1}, \cdots, E_{h-1}^{1} ; E_{h+1}^{1}, \cdots, E_{k}^{1} .
$$

In the next rank we have to take account only of the $x$-derivates of $E_{0}^{1}$ to $E_{h-1}^{1}$, the $y$-derivates of $E_{h+1}^{1}$ to $E_{k}^{1}$. For the only $y$-derivate not included among these is $D_{y} E_{h-1}^{1}$, which is the same as $D_{x} E_{h}^{1}$, and is therefore equal to $D_{x}\left(a_{0} E_{0}^{1}+a_{1} E_{1}^{1}+\cdots+a_{h-1} E_{h-1}^{1}\right)$; that is, it is a linear function of those 
$x$-derivates that have been taken into account. Similarly, the only $x$-derivate apparently neglected is

$$
\begin{aligned}
D_{x} E_{h+1}^{1} & \equiv D_{y} E_{h}^{1} \\
& \equiv D_{y}\left(a_{0} E_{0}^{1}+a_{1} E_{1}^{1}+\cdots+a_{h-1} E_{h-1}^{1}\right) \\
& \equiv D_{x}\left(a_{0} E_{1}^{1}+a_{1} E_{2}^{1}+\cdots+a_{h-1} E_{h}^{1}\right) .
\end{aligned}
$$

Now the $x$-derivates of $E_{1}^{1}, E_{2}^{1}, \cdots, E_{h-1}^{1}$ have been explicitly taken into account; and it has just been shown that $D_{x} E_{h}^{1}$ depends on these. Consequently no derivate has been neglected; all the derivates of the next rank are obtained by means of these $h x$-derivates and $k-h y$-derivates; their number is therefore $k$. A precisely similar proof applies to the equations of the next rank, and so on. Thus if all the equations become stationary, they remain stationary. The diagram now presents such an appearance as the following, for which the system of equations is that proceeding from the prime equation

$$
\begin{aligned}
& E=z_{0}^{8}+2 z_{1}^{8}-4 z_{2}^{8}+z_{3}^{8}+z_{4}^{8}+2 z_{5}^{8}-4 z_{6}^{8}+z_{7}^{8}+z_{8}^{8}+z_{0}^{7}-z_{3}^{7}+z_{4}^{7}-z_{7}^{7} \\
& +z_{0}^{6}-z_{1}^{6}+z_{4}^{6}-z_{5}^{6}+(z)^{5}+(z)^{4}+(z)^{3}=0 . \\
& E \text {. . . . of degree 8, } \\
& \begin{array}{lll}
E_{0}^{1} & E_{1}^{1} & \cdot
\end{array} \\
& \begin{array}{llll}
E_{0}^{2} & E_{1}^{2} & E_{2}^{2} & \text {. of degree } 6,
\end{array}
\end{aligned}
$$

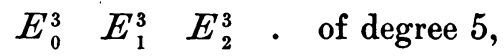

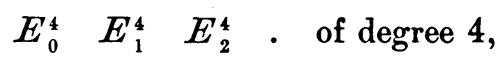

$$
\begin{aligned}
& \begin{array}{lllll}
E_{0}^{5} & E_{1}^{5} & E_{2}^{5} & \text {. of degree 3, }
\end{array} \\
& \begin{array}{lllll}
E_{0}^{6} & E_{1}^{6} & E_{2}^{6} & \text {. of degree } 2 .
\end{array}
\end{aligned}
$$

\section{The theorem of ascent.}

12. It is a simple matter to write down, beginning with the lowest terms, the general equation of a curve for which a given system of equations is satisfied. The converse operation, that of determining the equations satisfied by the coefficients of given curves that pass through the origin, though possibly lengthy, is simple enough, theoretically. As regards the coefficients of the lower terms, the equations can be found by a direct process; but for the higher terms, the process of ascent is more satisfactory. This is directly derived from a theorem now to be proved, but before entering upon this, it will be shown that two curves $u, v$ with a k-point at the origin satisfy either $k-1$ or $k$ equations of degree $k$, and $k-2, k-1$, or $k$ equations of degree $k+1$. 
Since every $z$ with index $<k$ is zero, the equations of degree $k$ are of the type

$$
\lambda_{0} z_{0}^{k}+\lambda_{1} z_{1}^{k}+\cdots+\lambda_{k} z_{k}^{k}=0 .
$$

Let the given curves be

$$
\begin{aligned}
& u=a_{0}^{k} x^{k}+a_{1}^{k} x^{k-1} y+\cdots+u_{k+1}+\cdots=0, \\
& v=b_{0}^{k} x^{k}+b_{1}^{k} x^{k-1} y+\cdots+v_{k+1}+\cdots=0,
\end{aligned}
$$

then the $\lambda$ 's are subject to the conditions

$$
\begin{aligned}
& \lambda_{0} a_{0}^{k}+\lambda_{1} a_{1}^{k}+\cdots+\lambda_{k} a_{k}^{k}=0, \\
& \lambda_{0} b_{0}^{k}+\lambda_{1} b_{1}^{k}+\cdots+\lambda_{k} b_{k}^{k}=0 .
\end{aligned}
$$

Hence the general equation of degree $k$ is

$$
\left|\begin{array}{ccc}
z_{0}^{k} & z_{1}^{k} & \lambda_{2} z_{2}^{k}+\cdots+\lambda_{k} z_{k}^{k} \\
a_{0}^{k} & a_{1}^{k} & \lambda_{2} a_{2}^{k}+\cdots+\lambda_{k} a_{k}^{k} \\
b_{0}^{k} & b_{1}^{k} & \lambda_{2} b_{2}^{k}+\cdots+\lambda_{k} b_{k}^{k}
\end{array}\right|=0,
$$

where the $\lambda$ 's are arbitrary. There are therefore $k-1$ equations of degree $k$, obtained by equating to zero the coefficients of the different $\lambda$ 's; these equations are given by any $k-1$ independent determinants of the set

$$
\left\|\begin{array}{ccccc}
z_{0}^{k} & z_{1}^{k} & z_{2}^{k} & \cdots & z_{k}^{k} \\
a_{0}^{k} & a_{1}^{k} & a_{2}^{k} & \cdots & a_{k}^{k} \\
b_{0}^{k} & b_{1}^{k} & b_{2}^{k} & \cdots & b_{k}^{k}
\end{array}\right\| .
$$

If however the tangents to $u, v$ at the origin are the same, so that

$$
a_{0}^{k}: a_{1}^{k}: a_{2}^{k}: \cdots=b_{0}^{k}: b_{1}^{k}: b_{2}^{k}: \cdots,
$$

the $\lambda$ 's are subject to only one condition, namely,

$$
\lambda_{0} a_{0}^{k}+\lambda_{1} a_{1}^{k}+\cdots+\lambda_{k} a_{k}^{k}=0,
$$

and the equations are those given by

that is,

$$
\left\|\begin{array}{llll}
z_{0}^{k} & z_{1}^{k} & \cdots & z_{k}^{k} \\
a_{0}^{k} & a_{1}^{k} & \cdots & a_{k}^{k}
\end{array}\right\|=0,
$$

$$
z_{0}^{k}: z_{1}^{k}: z_{2}^{k}: \cdots=a_{0}^{k}: a_{1}^{k}: a_{2}^{k}: \cdots,
$$

$k$ equations which express simply that the tangents are given.

If some of the $b$ 's are proportional to some of the $a$ 's, the equations remain $k-1$ in number. Every determinant can be written so as to contain one 
column in which the $b$ is not this same multiple of the $a$. The determinants are then of the two types

$$
\left|\begin{array}{rrr}
z_{0}^{k} & z_{3}^{k} & z_{4}^{k} \\
a_{0}^{k} & a_{3}^{k} & a_{4}^{k} \\
b_{0}^{k} & b_{3}^{k} & b_{4}^{k}
\end{array}\right|=0, \quad\left|\begin{array}{rrr}
z_{0}^{k} & z_{1}^{k} & z_{2}^{k} \\
a_{0}^{k} & a_{1}^{k} & a_{2}^{k} \\
b_{0}^{k} & \mu a_{1}^{k} & \mu a_{2}^{k}
\end{array}\right|=0,
$$

of which the second reduces to $z_{1}^{k}: a_{1}^{k}=z_{2}^{k}: a_{2}^{k}$. Thus the case is in no way exceptional.

No values for the $a$ 's and $b$ 's can make the number of equations exceed $k$, inasmuch as $k+1$ equations of degree $k$ would give for every $z^{k}$ the value zero, thus determining the multiple point on the curve as of order $k+1$. Hence the number of equations of degree $k$ is $k-1$ in the general case, $k$ if the curves have all their tangents in common.

It is now to be determined how many equations there are of degree $k+1$. If there are $k-1$ equations of degree $k$, by means of these all $z^{k}$ s can be expressed in terms of two only; if these are $z_{0}^{k}$ and $z_{k}^{k}$, then any equation of degree $k+1$ is

$$
\mu_{0} z_{0}^{k+1}+\mu_{1} z_{1}^{k+1}+\cdots+\mu_{k+1} z_{k+1}^{k+1}+a z_{0}^{k}+\beta z_{k}^{k}=0,
$$

where the $\mu$ 's are subject to the conditions

$$
\begin{aligned}
& \mu_{0} a_{0}^{k+1}+\mu_{1} a_{1}^{k+1}+\cdots+\mu_{k+1} a_{k+1}^{k+1}+a a_{0}^{k}+\beta a_{k}^{k}=0, \\
& \mu_{0} b_{0}^{k+1}+\mu_{1} b_{1}^{k+1}+\cdots+\mu_{k+1} b_{k+1}^{k+1}+a b_{0}^{k}+\beta b_{k}^{k}=0 .
\end{aligned}
$$

The $x$ - and $y$-derivates of these give other equations to be satisfied, namely

$$
\begin{aligned}
& \mu_{0} z_{0}^{k}+\mu_{1} z_{1}^{k}+\cdots+\mu_{k} z_{k}^{k}=0, \\
& \mu_{1} z_{0}^{k}+\mu_{2} z_{1}^{k}+\cdots+\mu_{k+1} z_{k}^{k}=0 ;
\end{aligned}
$$

hence the $\mu$ 's must satisfy

$$
\begin{aligned}
\mu_{0} a_{0}^{k}+\mu_{1} a_{1}^{k}+\cdots+\mu_{k} a_{k}^{k} & =0, \\
\mu_{1} a_{0}^{k}+\cdots+\mu_{k} a_{k-1}^{k}+\mu_{k+1} a_{k}^{k} & =0, \\
\mu_{0} b_{0}^{k}+\mu_{1} b_{1}^{k}+\cdots+\mu_{k} b_{k}^{k} & =0, \\
\mu_{1} b_{0}^{k}+\cdots+\mu_{k} b_{k-1}^{k}+\mu_{k+1} b_{k}^{k} & =0 .
\end{aligned}
$$

Thus the $k+4$ parameters $(k+2 \mu$ 's $, a, \beta)$ are subject to six equations, and so $k-2$ parameters are arbitrary. The number of equations of degree $k+1$ is therefore $k-2$ in general, though a linear relation connecting the six equations may increase this number. As however it is known that it cannot exceed the number of equations of degree $k$, it can only be $k-2$ or $k-1$. 
If however there are $k$ equations of degree $k$, every $z^{k}$ is known in terms of any one, e. g., $z_{0}^{k}$. Thus the general equation of degree $k+1$ is now

$$
\mu_{0} z_{0}^{k+1}+\mu_{1} z_{1}^{k+1}+\cdots+\mu_{k+1} z_{k+1}^{k+1}+a z_{0}^{k}=0,
$$

involving only $k+3$ parameters. The $\mu$ 's are subject to four equations only, since every $b^{k}$ is equal to the corresponding $a^{k}$. These are

$$
\begin{aligned}
\mu_{0} a_{0}^{k+1}+\mu_{1} a_{1}^{k+1}+\cdots+\mu_{k} a_{k}^{k+1}+\mu_{k+1} a_{k+1}^{k+1}+a a_{0}^{k} & =0, \\
\mu_{0} b_{0}^{k+1}+\mu_{1} b_{1}^{k+1}+\cdots+\mu_{k} b_{k}^{k+1}+\mu_{k+1} b_{k+1}^{k+1}+a a_{0}^{k} & =0, \\
\mu_{0} a_{0}^{k}+\mu_{1} a_{1}^{k}+\cdots+\mu_{k} a_{k}^{k} & =0, \\
\mu_{1} a_{0}^{k}+\cdots+\mu_{k} a_{k-1}^{k}+\mu_{k+1} a_{k}^{k} & =0 .
\end{aligned}
$$

The number of arbitrary parameters is therefore $k+3-4$, that is, $k-1$; the number of equations of degree $k+1$ is in general $k-1$, though a linear relation connecting the four equations may increase this number to $k$. As before, it is already known that it cannot exceed $k$.

13. The theorem of ascent is the following:

If two equations, satisfied by the coefficients. of two curves, have a common derivate, then they have a common source.

So much light is thrown on the important points in the proof by numerical examples, that it seems advisable to preface the formal discussion by two of these, relating to the two principal cases that present themselves.

(i) Let the curves be

$$
\begin{aligned}
& u=\cdots+u_{7}+x^{4} y^{2}+x^{3} y^{3}+x^{2} y^{4}+x^{5}-x^{3} y^{2}+x^{2} y^{3}-y^{5}+x^{4}+y^{4}=0, \\
& v=\cdots+v_{7}+x^{6}+x^{3} y^{3}+y^{6}+x^{5}+y^{5}+x^{2} y^{2}=0 ;
\end{aligned}
$$

these have at the origin quadruple points, with tangents that are different for the two curves. There are two equations of the fifth degree, namely,

$$
\begin{aligned}
& E_{1}=z_{1}^{5}-z_{5}^{5}-z_{0}^{4}+z_{2}^{4}=0, \\
& E_{2}=z_{0}^{5}-z_{4}^{5}-z_{0}^{4}-z_{2}^{4}=0 .
\end{aligned}
$$

Forming the derivates of these, we find

$$
\begin{array}{ll}
D_{x} E_{1}=z_{1}^{4}=0, & D_{y} E_{1}=z_{0}^{4}-z_{4}^{4}=0, \\
D_{x} E_{2}=z_{0}^{4}-z_{4}^{4}=0, & D_{y} E_{2}=-z_{3}^{4}=0 .
\end{array}
$$

Hence $D_{y} E_{1}=D_{x} E_{2}$. It is to be shown that an equation $E=0$ can be found, satisfied by $u$ and $v$, such that $E_{1}=D_{x} E, \quad E_{2}=D_{y} E$. 
It must be borne in mind that any equation can be modified by means of any of the derivates. Thus if $E$ be

$$
a_{0} z_{0}^{6}+\cdots+\beta_{0} z_{0}^{5}+\cdots+\gamma_{0} z_{0}^{4}+\cdots
$$

we can add to this $\lambda_{1} E_{1}+\lambda_{2} E_{2}$, so making two of the $\beta$ 's assume arbitrarily chosen values; and then we can modify the $\gamma$ 's in a similar manner. Since the origin is a quadruple point on both $u$ and $v$, every $z$ below $z^{4}$ is zero.

The values of $a$ are to be chosen so that the two derivates of $E$ may agree with $E_{1}$ and $E_{2}$; and if $\beta_{0}$ and $\beta_{5}$ are then made zero, as also $\gamma_{1}, \gamma_{3}, \gamma_{4}$, the whole equation can be written

$$
z_{1}^{6}-z_{5}^{6}+\beta_{1} z_{1}^{5}+\beta_{2} z_{2}^{5}+\beta_{3} z_{3}^{5}+\beta_{4} z_{4}^{5}+\gamma_{0} z_{0}^{4}+\gamma_{2} z_{2}^{4}=0 .
$$

The $x$-derivate of this, namely,

$$
z_{1}^{5}-z_{5}^{5}+\beta_{1} z_{1}^{4}+\beta_{2} z_{2}^{4}+\beta_{3} z_{3}^{4}+\beta_{4} z_{4}^{4},
$$

when modified by means of derivates, if necessary, is to be the same as

$$
z_{1}^{5}-z_{5}^{5}-z_{0}^{4}+z_{2}^{4} \text {. }
$$

Since $z_{1}^{4}=0, z_{3}^{4}=0$, and $z_{4}^{4}=z_{0}^{4}$, this requires $\beta_{2}=1, \beta_{4}=-1$.

Similarly

is to be the same as

$$
z_{0}^{5}-z_{4}^{5}+\beta_{1} z_{0}^{4}+\beta_{2} z_{1}^{4}+\beta_{3} z_{2}^{4}+\beta_{4} z_{3}^{4}
$$

$$
z_{0}^{5}-z_{4}^{5}-z_{0}^{4}-z_{2}^{4},
$$

and therefore $\beta_{1}=-1, \beta_{3}=-1$.

Comparison of the derivates gives no information about $\gamma_{0}$ and $\gamma_{2}$; thus the desired source is

$$
z_{1}^{6}-z_{5}^{6}-z_{1}^{5}+z_{2}^{5}-z_{3}^{5}-z_{4}^{5}+\gamma_{0} z_{0}^{4}+\gamma_{2} z_{2}^{4}=0 .
$$

Up to this point the result is applicable to any curves for which the given equations hold. The two parameters in the terms of degree 4 make it possible to apply the equation to any two such curves. For the two given curves the necessary values are $\gamma_{0}=2, \gamma_{2}=0$, and the desired source is the equation of the 6 th degree

$$
z_{1}^{6}-z_{5}^{6}-z_{1}^{5}+z_{2}^{5}-z_{3}^{5}-z_{4}^{5}+2 z_{0}^{4}=0 .
$$

The set of equations is in this case regular, and the diagram is

$$
\begin{aligned}
& E \cdot \cdot \cdot \cdot \cdot \text { of degree } 6, \\
& E_{0}^{1} E_{1}^{1} \cdot \cdot \cdot \text { of degree } 5, \\
& E_{0}^{2} E_{1}^{2} E_{2}^{2} \cdot . \quad \text { of degree } 4, \\
& E_{0}^{3} E_{1}^{3} E_{2}^{3} E_{3}^{3} \cdot \quad \text { of degree } 3 .
\end{aligned}
$$


The presence, in the source determined without reference to the particular curves, of two arbitrary parameters in the terms of lowest degree $k$, is what characterizes the solution.

(ii) For the curves

$$
\begin{aligned}
u=\cdots+ & x^{6}+x^{4} y^{2}-x^{3} y^{3}+y^{6}+10 x^{5}+5 x^{4} y+20 x^{3} y^{2}+5 x^{2} y^{3}+10 x y^{4} \\
& +5 y^{5}+5 x^{4}+8 x^{3} y+3 x^{2} y^{2}+x y^{3}+2 x^{3}+7 x^{2} y+7 x y^{2}+2 y^{3}=0 \\
v=\cdots+ & 6 x^{6}-5 x^{5} y+x^{4} y^{2}-x^{3} y^{3}-10 x^{2} y^{4}-5 x y^{5}-6 y^{6}-8 x^{5}-48 x^{4} y \\
& -12 x^{3} y^{2}+x^{2} y^{3}+8 x y^{4}+3 y^{5}+x^{4}-4 x^{3} y-4 x^{2} y^{2}+4 x y^{3}+2 y^{4} \\
& +2 x^{3}+7 x^{2} y+7 x y^{2}+2 y^{3}=0
\end{aligned}
$$

there are satisfied two equations of the fifth degree with all their derivates. These are

$$
\begin{gathered}
E_{1}=32 z_{0}^{5}-56 z_{1}^{5}+88 z_{2}^{5}-144 z_{3}^{5}+252 z_{4}^{5}-466 z_{5}^{5} \\
+37 z_{0}^{4}+5 z_{1}^{4}+7 z_{2}^{4}+5 z_{3}^{4}-169 z_{4}^{4}-\frac{1521}{2} z_{0}^{3}=0, \\
E_{2}=-56 z_{0}^{5}+88 z_{1}^{5}-144 z_{2}^{5}+252 z_{3}^{5}-466 z_{4}^{5}+893 z_{5}^{5} \\
-181 z_{0}^{4}+6 z_{1}^{4}+8 z_{2}^{4}+10 z_{3}^{4}+142 z_{4}^{4}+1379 z_{0}^{3}=0 .
\end{gathered}
$$

The identity of the $y$-derivate of the first with the $x$-derivate of the second appears when the terms of the third degree are modified by means of the values for $z_{0}^{3}, z_{1}^{3}, z_{2}^{3}, z_{3}^{3}$ given by the next derivates. These derivates,

$$
\begin{aligned}
& D_{x}^{2} E_{1}=32 z_{0}^{3}-56 z_{1}^{3}+88 z_{2}^{3}-144 z_{3}^{3}=0, \\
& D_{x} D_{y} E_{1}=D_{x}^{2} E_{2}=-56 z_{0}^{3}+88 z_{1}^{3}-144 z_{2}^{3}+252 z_{3}^{3}=0, \\
& D_{y}^{2} E_{1}=D_{x} D_{y} E_{2}=88 z_{0}^{3}-144 z_{1}^{3}+252 z_{2}^{3}-466 z_{3}^{3}=0, \\
& D_{y}^{2} E_{2}=-144 z_{0}^{3}+252 z_{1}^{3}-466 z_{2}^{3}+893 z_{3}^{3}=0,
\end{aligned}
$$

are equivalent to three only, for the result of multiplying by $2,7,7,2$ and adding vanishes identically; solving, we have $z_{0}^{3}: z_{1}^{3}: z_{2}^{3}: z_{3}^{3}=2: 7: 7: 2$.

By means of these values, the derivates of $E_{1}, E_{2}$ can be written

$$
\begin{aligned}
& D_{x} E_{1}=32 z_{0}^{4}-56 z_{1}^{4}+88 z_{2}^{4}-144 z_{3}^{4}+252 z_{4}^{4}+84 z_{0}^{3}=0, \\
& D_{y} E_{1}=D_{x} E_{2}=-56 z_{0}^{4}+88 z_{1}^{4}-144 z_{2}^{4}+252 z_{3}^{4}-466 z_{4}^{4}-122 z_{0}^{3}=0, \\
& D_{y} E_{2}=88 z_{0}^{4}-144 z_{1}^{4}+252 z_{2}^{4}-466 z_{3}^{4}+893 z_{4}^{4}+211 z_{0}^{3}=0 .
\end{aligned}
$$


The highest terms in a possible source for these two can be written down at once, and the equation of the source can be taken as

$$
\begin{aligned}
E=32 z_{0}^{6}-56 z_{1}^{6}+88 z_{2}^{6}-144 z_{3}^{6}+252 & z_{4}^{6}-466 z_{5}^{6}+893 z_{6}^{6} \\
& +\beta_{0} z_{0}^{5}+\cdots+\left(z_{0}^{4}, z_{1}^{4}, \cdots\right)+\kappa z_{0}^{3}=0 .
\end{aligned}
$$

The two derivates of this are to agree with the given equations; hence

must agree with

$$
\begin{aligned}
& \beta_{0} z_{0}^{4}+\beta_{1} z_{1}^{4}+\cdots+\beta_{4} z_{4}^{4}, \\
& \beta_{1} z_{0}^{4}+\beta_{2} z_{1}^{4}+\cdots+\beta_{5} z_{4}^{4},
\end{aligned}
$$

and

$$
37 z_{0}^{4}+5 z_{1}^{4}+7 z_{2}^{4}+5 z_{3}^{4}-169 z_{4}^{4}
$$

$$
-181 z_{0}^{4}+6 z_{1}^{4}+8 z_{2}^{4}+10 z_{3}^{4}+142 z_{4}^{4}
$$

with the help of the derivates of the fourth degree. This yields the equations

$$
\begin{array}{lrr}
\beta_{0}= & 37+32 \lambda_{1}-56 \lambda_{2}+88 \lambda_{3}, \\
\beta_{1}= & 5-56 \lambda_{1}+88 \lambda_{2}-144 \lambda_{3}= & -181+32 \lambda_{1}^{\prime}-56 \lambda_{2}^{\prime}+88 \lambda_{3}^{\prime}, \\
\beta_{2}= & 7+88 \lambda_{1}-144 \lambda_{2}+252 \lambda_{3}= & 6-56 \lambda_{1}^{\prime}+88 \lambda_{2}^{\prime}-144 \lambda_{3}^{\prime}, \\
\beta_{3}= & 5-144 \lambda_{1}+252 \lambda_{2}-466 \lambda_{3}= & 8+88 \lambda_{1}^{\prime}-144 \lambda_{2}^{\prime}+252 \lambda_{3}^{\prime}, \\
\beta_{4}=-169+252 \lambda_{1}-466 \lambda_{2}+893 \lambda_{3}= & 10-144 \lambda_{1}^{\prime}+252 \lambda_{2}^{\prime}-466 \lambda_{3}^{\prime}, \\
\beta_{5} & =142+252 \lambda_{1}^{\prime}-466 \lambda_{2}^{\prime}+893 \lambda_{3}^{\prime} .
\end{array}
$$

The four equations in the $\lambda$ 's and $\lambda$ 's, obtained by equating the two values for $\beta_{1}, \beta_{2}, \beta_{3}$ and $\beta_{4}$, are equivalent to three only, on account of the relation already noted in forming the derivates, which holds also for the numerical part of the equations now in question. Moreover, any two relations in the $\beta$ 's can be imposed arbitrarily, inasmuch as the source can be modified by the addition of linear multiples of $E_{1}$ and $E_{2}$. For simplicity, let these relations be such that $\lambda_{2}^{\prime}=0, \lambda_{3}^{\prime}=0$; the three equations then give

$$
\lambda_{1}=-\frac{837}{80} \frac{5}{8}+\frac{7}{8} A, \quad \lambda_{2}=-\frac{10987}{80}+\frac{7}{8} A, \quad \lambda_{3}=-1 \frac{67}{4} \frac{7}{6}+\frac{1}{4} A,
$$

where $A$ is written for $-4 \lambda_{1}^{\prime}$; and the $\beta$ 's are consequently

$$
\begin{array}{lll}
\beta_{0}=688 \frac{1}{2}+A, & \beta_{2}=6+14 A, & \beta_{4}=10+36 A, \\
\beta_{1}=-181-8 A, & \beta_{3}=8-22 A, & \beta_{5}=142-63 A .
\end{array}
$$


The source can now be written

$$
\begin{aligned}
& 32 z_{0}^{6}-56 z_{1}^{6}+88 z_{2}^{6}- 144 z_{3}^{6}+252 z_{4}^{6}-466 z_{5}^{6}+893 z_{6}^{6} \\
&+\left(688 \frac{1}{2} z_{0}^{5}-181 z_{1}^{5}+6 z_{2}^{5}+8 z_{3}^{5}+10 z_{4}^{5}+142 z_{5}^{5}\right) \\
&+A\left(z_{0}^{5}-8 z_{1}^{5}+14 z_{2}^{5}-22 z_{3}^{5}+36 z_{4}^{5}-63 z_{5}^{5}\right) \\
& \quad+\gamma_{0} z_{0}^{4}+\gamma_{4} z_{4}^{4}+\kappa z_{0}^{3}=0,
\end{aligned}
$$

in which the terms in $z^{4}$ have been modified by the addition of linear multiples of the three independent derivates of the fourth degree, chosen so as to make $\gamma_{1}=0, \gamma_{2}=0, \gamma_{3}=0$; and comparing the derivates of this, with $E_{1}$ and $E_{2}$, increased by $\lambda_{1} D_{x} E_{1}+\lambda_{2} D_{x} E_{2}+\lambda_{3} D_{y} E_{2}$ and $-\frac{1}{4} A D_{x} E_{1}$, we find that $\gamma_{0} z_{0}^{3}$ must agree with $-12_{2}^{2} 1 z_{0}^{3}+\lambda_{1} 84 z_{0}^{3}-\lambda_{2} 122 z_{0}^{3}+\lambda_{3} 211 z_{0}^{3}$, and $\gamma_{4} z_{3}^{3}$ with $1379 z_{0}^{3}-1 A 84 z_{0}^{3}$.

The first gives

$$
\begin{aligned}
\gamma_{0}=-\frac{1521}{2}+\left(-\frac{8}{8} \frac{7}{8} \frac{5}{2}+\frac{7}{8} A\right) 84 & +\left(\frac{10987}{80}-\frac{7}{8} A\right) 122 \\
& +\left(-\frac{16}{4} \frac{77}{7}+\frac{1}{4} A\right) 211=-\frac{6581}{4}+\frac{89}{2} A ;
\end{aligned}
$$

and the second, by help of the relation $z_{0}^{3}=z_{3}^{3}$, gives $\gamma_{4}=1379-21 \mathrm{~A}$.

Thus the desired source is of the form

$$
\begin{aligned}
32 z_{0}^{6}- & 56 z_{1}^{6}+88 z_{2}^{6}-144 z_{3}^{6}+252 z_{4}^{6}-466 z_{5}^{6}+893 z_{6}^{6} \\
& +\left(688 \frac{1}{2} z_{0}^{5}-181 z_{1}^{5}+6 z_{2}^{5}+8 z_{3}^{5}+10 z_{4}^{5}+142 z_{5}^{5}-\frac{65}{4} z_{0}^{4}+1379 z_{4}^{4}\right) \\
& +A\left(z_{0}^{5}-8 z_{1}^{5}+14 z_{2}^{5}-22 z_{3}^{5}+36 z_{4}^{5}-63 z_{5}^{5}+\frac{39}{2} z_{0}^{4}-21 z_{4}^{4}\right)+\kappa z_{0}^{3}=0 .
\end{aligned}
$$

As before, this involves two parameters, which must be determined by means of the two given curves. The values found are $A=\frac{1}{2}, \kappa=-11$, so that the two given equations are derived from the source

$$
\begin{aligned}
32 z_{0}^{6}-56 z_{1}^{6}+ & 88 z_{2}^{6}-144 z_{3}^{6}+252 z_{4}^{6}-466 z_{5}^{6}+893 z_{6}^{6}+689 z_{0}^{5}-185 z_{1}^{5} \\
& +13 z_{2}^{5}-3 z_{3}^{5}+28 z_{4}^{5}+\frac{2}{2} \frac{1}{2} z_{5}^{5}-\frac{3}{2} 2 \underline{2} z_{0}^{4}+\frac{27}{2} z_{4} z_{4}^{4}-11 z_{0}^{3}=0
\end{aligned}
$$

The equations in this example are

$$
\begin{array}{llllll}
E & \cdot & \cdot & \cdot & . & \text { of degree } 6, \\
E_{0}^{1} & E_{1}^{1} & \cdot & \cdot & . & \text { of degree } 5, \\
E_{0}^{2} & E_{1}^{2} & E_{2}^{2} & . & \text { of degree } 4, \\
E_{0}^{3} & E_{1}^{3} & E_{2}^{3} & . & \text { of degree } 3 ;
\end{array}
$$

the solution is characterized by the presence of two parameters in the source, so far as determined without reference to the particular curves, but there is now 
the difference that on y one of these is in the terms of degree $k$; the first arbitrary parameter appears in connection with the suspension of the law of unit increase in the number of the equations obtained at the successive stages of the derivation.

14. The general algebraic proof that if two equations have a common derivate they have a common source for two curves, depends on obtaining the equation of a source involving two arbitrary parameters.

The two given equations of degree $p-1$ can be reduced to the form

$$
\begin{gathered}
E_{1}=a_{0} z_{0}^{p-1}+a_{1} z_{1}^{p-1}+a_{2} z_{2}^{p-1}+\cdots+a_{p-1} z_{p-1}^{p-1}+b_{0} z_{0}^{p-2}+b_{1} z_{1}^{p-2} \\
+\cdots+c_{0} z_{0}^{p-3}+\cdots=0, \\
\begin{array}{c}
E_{2}=a_{1} z_{0}^{p-1}+a_{2} z_{1}^{p-1}+a_{3} z_{2}^{p-1}+\cdots+a_{p} z_{p-1}^{p-1}+b_{1} z_{0}^{p-2}+b_{2} z_{1}^{p-2} \\
+\cdots+c_{1} z_{0}^{p-3}+\cdots=0,
\end{array}
\end{gathered}
$$

in which the agreement of $D_{y} E_{1}$ with $D_{x} E_{2}$ is obvious. For if the second is given as

$$
E^{\prime}=a_{1}^{\prime} z_{0}^{p-1}+a_{2}^{\prime} z_{1}^{p-1}+\cdots+a_{p}^{\prime} z_{p-1}^{p-1}+b_{1}^{\prime} z_{0}^{p-2}+b_{2}^{\prime} z_{1}^{p-2}+\cdots+c_{1}^{\prime} z_{0}^{p-3}+\cdots=0,
$$

the fact that the four derivates reduce to three shows that there is a linear relation which may be written

that is,

$$
\lambda D_{x} E_{1}+\mu D_{y} E_{\mathrm{l}}+\lambda^{\prime} D_{x} E^{\prime}+\mu^{\prime} D_{y} E^{\prime}=0,
$$

$$
D_{x}\left(\lambda E_{1}+\lambda^{\prime} E^{\prime}\right)+D_{y}\left(\mu E_{1}+\mu^{\prime} E^{\prime}\right)=0 ;
$$

thus if we take instead of the given equations these two linear combinations we have the desired form. Even if the given equations are specialized so that they yield only two derivates instead of three, they can be written in this form; the coefficients are now subject to the conditions obtained by expressing that the three equations

$$
a_{i} z_{0}^{p-2}+\cdots+b_{i} z_{0}^{p-3}+\cdots+c_{i} z_{0}^{p-4}+\cdots=0 \quad(i=0,1,2)
$$

are equivalent to two only, namely, the vanishing of the determinants

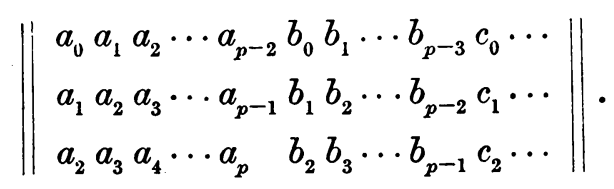


If $\left(a_{h}\right)^{q}$ be written for $a_{h} z_{0}^{q}+a_{h+1} z_{1}^{q}+a_{h+2} z_{2}^{q}+\cdots+a_{h+q} z_{q}^{q}$, the given equations become

$$
\begin{aligned}
& E_{1}=\left(a_{0}\right)^{p-1}+\left(b_{0}\right)^{p-2}+\left(c_{0}\right)^{p-3}+\cdots=0, \\
& E_{2}=\left(a_{1}\right)^{p-1}+\left(b_{1}\right)^{p-2}+\left(c_{1}\right)^{p-3}+\cdots=0 .
\end{aligned}
$$

Let the source to be found be written

$$
E=\left(a_{0}\right)^{p}+\left(\beta_{0}\right)^{p-1}+\left(\gamma_{0}\right)^{p-2}+\cdots+\left(\tau_{0}\right)^{k+1}+()^{k}=0 .
$$

For the determination of the coefficients we have the equations obtained by expressing the identity of the two derivates of $E$ with the given equations, modified by the addition of multiples of lower derivates. These will give for $(a),(\beta)$, $(\gamma)$, etc., values involving a certain number of arbitrary parameters. Inasmuch however as the source $E$ may itself be modified in the same manner, its most general expression must involve a certain number of non-significant parameters. The terms $\left(\beta_{0}\right)^{p-1}$, for instance, may be modified by the addition of $B_{1} E_{1}+B_{2} E_{2}$; to the $B$ 's arbitrary values may be assigned; we may, e. g., if we choose, make $\beta_{0}$ and $\beta_{p-1}=0$. The $\beta$ 's, then, must involve in their expression two nonsignificant parameters; and if they involve only two in all, both are non-significant, and the $\beta$ 's are looked upon as absolutely determinate.

The agreement of the two derivates of $E$ with the given equations requires only that $D_{x} E$ and $D_{y} E$ shall be linear functions of $E_{1}$ and $E_{2}$, that is, that

$$
D_{x} E=\lambda_{1} E_{1}+\lambda_{2} E_{2}, \quad D_{y} E=\lambda_{1}^{\prime} E_{1}+\lambda_{2}^{\prime} E_{2} .
$$

Hence we must have

$$
\begin{aligned}
a_{0}=\lambda_{1} a_{0}+\lambda_{2} a_{1}, & \\
a_{i}=\lambda_{1} a_{i}+\lambda_{2} a_{i+1} & =\lambda_{1}^{\prime} a_{i-1}+\lambda_{2}^{\prime} a_{i} \quad(i=1, \cdots, p-1), \\
a_{p} & =\lambda_{1}^{\prime} a_{p-1}+\lambda_{2}^{\prime} a_{p} .
\end{aligned}
$$

The equations for $\lambda_{1}, \lambda_{2}, \lambda_{1}^{\prime}, \lambda_{2}^{\prime}$ are therefore

$$
\lambda_{1}^{\prime} a_{i-1}+\left(\lambda_{2}^{\prime}-\lambda_{1}\right) a_{i}-\lambda_{2} a_{i+1}=0 \quad(i=1, \cdots, p-1),
$$

where $p$ is necessarily $\geqq 4$. Unless the determinants of the third order

$$
\left\|\begin{array}{llllll}
a_{0} & a_{1} & a_{2} & a_{3} & \cdots & a_{p-2} \\
a_{1} & a_{2} & a_{3} & a_{4} & \cdots & a_{p-1} \\
a_{2} & a_{3} & a_{4} & a_{5} & \cdots & a_{p}
\end{array}\right\| .
$$

all vanish, these equations give

$$
\lambda_{1}^{\prime}=0, \quad \lambda_{2}^{\prime}=\lambda_{1}, \quad \lambda_{2}=0,
$$


from which $\left(a_{0}\right)^{p}=\lambda_{1}\left(a_{0}\right)^{p}$, where the $\lambda_{1}$ may of course be taken unity. In this case $D_{x} E=E_{1}, D_{y} E=E_{2}$.

If however these determinants do vanish, that is, if

$$
a_{i+1}=\eta_{1} a_{i-1}+\eta_{2} a_{i} \quad(i=1, \cdots, p-1),
$$

the equations reduce to

$$
\left(\lambda_{1}^{\prime}-\eta_{1} \lambda_{2}\right) a_{i-1}+\left(\lambda_{2}^{\prime}-\lambda_{1}-\eta_{2} \lambda_{2}\right) a_{i}=0 \quad(i=1, \cdots, p-1),
$$

from which

$$
\lambda_{1}^{\prime}=\eta_{1} \lambda_{2}, \quad \lambda_{2}^{\prime}=\lambda_{1}+\eta_{2} \lambda_{2}
$$

Hence

$$
a_{i}=\lambda_{1} a_{i}+\lambda_{2} a_{i+1}, \quad(i=0, \cdots, p),
$$

where $\lambda_{1}, \lambda_{2}$ are arbitrary parameters, and $a_{p+1}$ is written for $\eta_{1} a_{p-1}+\eta_{2} a_{p}$.

Thus if the two given equations yield only two derivates the terms of highest degree in the source can be written $A_{1}\left(a_{0}\right)^{p}+A_{2}\left(a_{1}\right)^{p}$; this special case is considered in $\S 19$. In the general case the coefficients $a$ are determined without ambiguity, and the source is

$$
E=\left(a_{0}\right)^{p}+\left(\beta_{0}\right)^{p-1}+\left(\gamma_{0}\right)^{p-2}+\cdots=0
$$

15. The next step in the comparison of the two derivates of $E$ with $E_{1}$ and $E_{2}$ shows that the $\beta$ 's must satisfy

$\beta_{0}=b_{0}+\lambda_{1} a_{0}+\lambda_{2} a_{1}+\lambda_{3} a_{2}$,

$\beta_{i}=b_{i}+\lambda_{1} a_{i}+\lambda_{2} a_{i+1}+\lambda_{3} a_{i+2}=b_{i}+\lambda_{1}^{\prime} a_{i-1}+\lambda_{2}^{\prime} a_{i}+\lambda_{3}^{\prime} a_{i+1} \quad(i=1, \cdots, p-2)$,

$\beta_{p-1}$

$$
=b_{p-1}+\lambda_{1}^{\prime} a_{p-2}+\lambda_{2}^{\prime} a_{p-1}+\lambda_{3}^{\prime} a_{p},
$$

where the $\lambda$ 's and $\lambda$ 's are to be determined. The equations obtained from the double values for $\beta_{1}, \cdots, \beta_{p-2}$ give

Unless

$$
\lambda_{1}^{\prime} a_{i-1}+\left(\lambda_{2}^{\prime}-\lambda_{1}\right) a_{i}+\left(\lambda_{3}^{\prime}-\lambda_{2}\right) a_{i+1}-\lambda_{3} a_{i+2}=0 \quad(i=1, \cdots, p-2) .
$$

$$
\left\|\begin{array}{cccc}
a_{0} & a_{1} & \cdots & a_{p-3} \\
a_{1} & a_{2} & \cdots & a_{p-2} \\
a_{2} & a_{3} & \cdots & a_{p-1} \\
a_{3} & a_{4} & \cdots & a_{p}
\end{array}\right\|=0
$$


(in which case the four derivates of degree $p-3$ reduce to three only) these equations give

from which

$$
\lambda_{1}^{\prime}=0, \quad \lambda_{2}^{\prime}=\lambda_{1}, \quad \lambda_{3}^{\prime}=\lambda_{2}, \quad \lambda_{3}=0,
$$

$$
\beta_{i}=b_{i}+\lambda_{1} a_{i}+\lambda_{2} a_{i+1} \quad(i=0, \cdots, p-1),
$$

where $\lambda_{1}, \lambda_{2}$ are arbitrary. The $\beta$ 's, however, involve two non-significant parameters, since, as already pointed out, the source may be modified by the addition of $B_{1} E_{1}$ and $B_{2} E_{2}$; we may therefore choose $\lambda_{1}, \lambda_{2}$ arbitrarily, for instance, $\lambda_{1}=0, \lambda_{2}=0$, values which have the advantage of simplifying the work, inasmuch as no multiples of the three derivates of $E_{1}$ and $E_{2}$ are now used. We have now $\beta_{0}=b_{0}$, etc., and the source is

$$
\left(a_{0}\right)^{p}+\left(b_{0}\right)^{p-1}+\left(\gamma_{0}\right)^{p-2}+\left(\delta_{0}\right)^{p-3}+\cdots=0 .
$$

The derivates of this, namely,

$$
\left(a_{i}\right)^{p-1}+\left(b_{i}\right)^{p-2}+\left(\gamma_{i}\right)^{p-3}+\left(\delta_{i}\right)^{p-4}+\cdots=0 \quad(i=0,1),
$$

are to agree with

$$
\left(a_{i}\right)^{p-1}+\left(b_{i}\right)^{p-2}+\left(c_{i}\right)^{p-3}+\left(d_{i}\right)^{p-4}+\cdots=0 \quad(i=0,1),
$$

with the help of the four derivates of degree $p-3$,

hence

$$
\left(a_{i}\right)^{p-3}+\left(b_{i}\right)^{p-4}+\cdots=0 \quad(i=0,1,2,3) ;
$$

$$
\begin{aligned}
& \gamma_{0}=c_{0}+\lambda_{1} a_{0}+\lambda_{2} a_{1}+\lambda_{3} a_{2}+\lambda_{4} a_{3}, \\
& \gamma_{i}=c_{i}+\lambda_{1} a_{i}+\lambda_{2} a_{i+1}+\lambda_{3} a_{i+2}+\lambda_{4} a_{i+3}=c_{i}+\lambda_{1}^{\prime} a_{i-1}+\lambda_{2}^{\prime} a_{i}+\lambda_{3}^{\prime} a_{i+1}+\lambda_{4}^{\prime} a_{i+2} \\
& (i=1, \cdots, p-3) \text {, } \\
& \gamma_{p-2} \\
& =c_{p-2}+\lambda_{1}^{\prime} a_{p-3}+\lambda_{2}^{\prime} a_{p-2}+\lambda_{3}^{\prime} a_{p-1}+\lambda_{4}^{\prime} a_{p} .
\end{aligned}
$$

Then the equations for the $\lambda$ 's and $\lambda^{\prime \prime}$ s are

$$
\begin{array}{r}
\lambda_{1}^{\prime} a_{i-1}+\left(\lambda_{2}^{\prime}-\lambda_{1}\right) a_{i}+\left(\lambda_{3}^{\prime}-\lambda_{2}\right) a_{i+1}+\left(\lambda_{4}^{\prime}-\lambda_{3}\right) a_{i+2}-\lambda_{4} a_{i+3}=0 \\
(i=1, \cdots, p-3) ;
\end{array}
$$

hence, unless

we find

$$
\left\|\begin{array}{llll}
a_{0} & a_{1} & \cdots & a_{p-4} \\
a_{1} & a_{2} & \cdots & a_{p-3} \\
a_{2} & a_{3} & \cdots & a_{p-2} \\
a_{3} & a_{4} & \cdots & a_{p-1} \\
a_{4} & a_{5} & \cdots & a_{p}
\end{array}\right\|=0
$$

$$
\lambda_{1}^{\prime}=0, \quad \lambda_{2}^{\prime}=\lambda_{1}, \quad \lambda_{3}^{\prime}=\lambda_{2}, \quad \lambda_{4}^{\prime}=\lambda_{3}, \quad \lambda_{4}=0 .
$$


The expressions for the $\gamma$ 's involve therefore the three parameters $\lambda_{1}, \lambda_{2}, \lambda_{3}$; since however there must be three non-significant parameters, we may choose $\lambda_{1}=0, \lambda_{2}=0, \lambda_{3}=0$. Then $\gamma_{0}=c_{0}, \gamma_{1}=c_{1}$, etc., and the source is

$$
E=\left(a_{0}\right)^{p}+\left(b_{0}\right)^{p-1}+\left(c_{0}\right)^{p-2}+\text { etc. } \cdots=0 \text {. }
$$

This argument is perfectly general; the coefficients in the terms of degree $p-m$ are linearly expressed in terms of the quantities

$$
\lambda_{1}, \lambda_{2}, \cdots, \lambda_{m+2} ; \lambda_{1}^{\prime}, \lambda_{2}^{\prime}, \cdots, \lambda_{m+2}^{\prime},
$$

given by the equations

$$
\begin{aligned}
& \lambda_{1}^{\prime} a_{i-1}+\left(\lambda_{2}^{\prime}-\lambda_{1}\right) a_{i}+\left(\lambda_{3}^{\prime}-\lambda_{2}\right) a_{i+1}+\cdots \\
& \quad+\left(\lambda_{m+2}^{\prime}-\lambda_{m+1}\right) a_{i+m}-\lambda_{m+2} a_{i+m+1}=0 \quad(i=1, \cdots, p-m-1) ;
\end{aligned}
$$

here there are $m+3$ quantities to be determined by means of $p-m-1$ equations. Leaving the terms of degree $k$ or $k+1$ to be considered separately, we have $p-m>k$, and $m<k-2$, consequently

$$
p-m-1>k-1, \quad m+2<k,
$$

that is, $p-m-1 \geqq m+3$. Every combination of $\lambda^{\prime}$ s and $\lambda^{\prime \prime}$ 's is therefore zero. Hence, unless

$$
\left\|\begin{array}{llll}
a_{0} & a_{1} & \cdots & a_{p-m-2} \\
a_{1} & a_{2} & \cdots & \vdots \\
\vdots & & & \vdots \\
a_{m+2} & a_{m+3} & \cdots & a_{p}
\end{array}\right\|=0,
$$

(that is, unless the $m+3$ derivates of degree $p-m-2$ reduce to $m+2$ of this degree), the coefficients of the terms of degree $p-m$ involve linearly parameters whose number, $m+1$, is precisely the number of non-significant parameters that must enter into these coefficients; these may all be taken zero, with the result that the coefficients of the terms of degree $p-m$ in the source are those immediately given by the terms of degree $p-m-1$ in the two given equations.

If now there is no reduction in the number of derivates at any stage, the scheme of equations is regular, and there are therefore $k-1$ equations of degree $k$, by means of which the values of $z_{0}^{k}, z_{1}^{k}, \ldots, z_{k}^{k}$ are all expressed in terms of any two, e. g., of $z_{0}^{k}$ and $z_{k}^{k}$. The terms of degree $k$ in the source can then be written $A_{1} z_{0}^{k}+A_{2} z_{k}^{k}$, and as no information is given about $A_{1}, A_{2}$ by comparison with the derivates, since these terms disappear on derivation, these are two arbitrary parameters entering into the equation of the source,

$$
E=\left(a_{0}\right)^{p}+\left(b_{0}\right)^{p-1}+\left(c_{0}\right)^{p-2}+\cdots+\left(k_{0}\right)^{k+1}+A_{1} z_{0}^{k}+A_{2} z_{k}^{k}=0 .
$$

16. If there are $k$ equations of degree $k$, so that $z_{0}^{k}, z_{1}^{k}, \cdots, z_{k}^{k}$ are all expressed in terms of any one, the scheme is not strictly regular, even though it be regular 
up to this point. In this case $p=2 k-1$. The terms of degree $k$ in the source are now reducible to $A z_{0}^{k}$, involving only one parameter. But the equations for $\tau_{0}, \tau_{1}, \cdots, \tau_{k+1}$ are

$$
\begin{array}{ccc}
\tau_{0}=t_{0}+\lambda_{1} a_{0}+\cdots+\lambda_{k} a_{k-1} & \\
\tau_{i}=t_{i}+\lambda_{1} a_{i}+\cdots+\lambda_{k} a_{i+k-1} & =t_{i}+\lambda_{1}^{\prime} a_{i-1} & +\cdots+\lambda_{k}^{\prime} a_{i+k-2} \\
& (i=1, \cdots, k), \\
\tau_{k+1} & =t_{k+1}+\lambda_{1}^{\prime} a_{k}+\cdots+\lambda_{k}^{\prime} a_{2 k-1} .
\end{array}
$$

Hence we have for the $\lambda$ 's and $\lambda^{\prime \prime}$ s the $k$ equations :

$$
\lambda_{1}^{\prime} a_{i-1}+\left(\lambda_{2}^{\prime}-\lambda_{1}\right) a_{i}+\cdots+\left(\lambda_{k}^{\prime}-\lambda_{k-1}\right) a_{i+k-2}-\lambda_{k} a_{i+k-1}=0 \quad(i=1, \cdots, k),
$$

involving the $\lambda$ 's, etc., in $k+1$ combinations. By means of these equations the quantities $\lambda^{\prime}$ are expressed in terms of the $k$ parameters $\lambda_{1}, \ldots, \lambda_{k}$. The terms $\left(\tau_{0}\right)^{k+1}$ in the source involve $k-1$ non-significant parameters, since there are $k-1$ derivates of degree $k+1$, and accordingly there is one significant parameter involved in these terms. As before, there are two arbitrary parameters in the equation of the source, which is now

$$
E=\left(a_{0}\right)^{p}+\left(b_{0}\right)^{p-1}+\cdots+\left(t_{0}\right)^{k+1}+A_{1} \bar{E}+A_{2} z_{0}^{k}=0,
$$

where $\bar{E}$ is of degree $k+1$.

17. Partial agreement of the derivates.-At every stage in the determination of the coefficients in the source the possibility of an exception presents itself. This exception depends on the existence of a relation among the $a$ 's, by means of which the $m+3$ derivates of degree $p-m-2$ are reduced to $m+2$. Let the terms in the source be $\left(\rho_{0}\right)^{p-m}$; the agreement of $\left(\rho_{0}\right)^{p-m-1}$ and $\left(\rho_{1}\right)^{p-m-1}$ with $\left(r_{0}\right)^{p-m-1}$ and $\left(r_{1}\right)^{p-m-1}$ gives the equations

$$
\begin{array}{ll}
\rho_{0}=r_{0}+\lambda_{1} a_{0}+\cdots+\lambda_{m+2} a_{m+1}, & \\
\rho_{i}=r_{i}+\lambda_{1} a_{i}+\cdots+\lambda_{m+2} a_{i+m+1}=r_{i}+\lambda_{1}^{\prime} a_{i-1}+\cdots+\lambda_{m+2}^{\prime} a_{i+m} \\
\rho_{p-m}=r_{p-m}+\lambda_{1}^{\prime} a_{p-m-1}+\cdots+\lambda_{m+2}^{\prime} a_{p} ; & (i=1, \cdots, p-m-1),
\end{array}
$$

the $\lambda$ 's and $\lambda$ 's are therefore determined by $p-m-1$ equations of the usual type,

$$
\begin{aligned}
\lambda_{1}^{\prime} a_{i-1}+\left(\lambda_{2}^{\prime}-\lambda_{1}\right) a_{i}+\cdots+\left(\lambda_{m+2}^{\prime}-\lambda_{m+1}\right) a_{i+m}-\lambda_{m+2} a_{i+m+1}=0 \\
(i=1, \cdots, p-m-1),
\end{aligned}
$$

in which the quantities appear in $m+3$ combinations. But by hypothesis

$$
a_{i+m+1}=\eta_{1} a_{i-1}+\eta_{2} a_{i}+\eta_{3} a_{i+1}+\cdots+\eta_{m+2} a_{i+m} \quad(i=1, \cdots, p-m-1) ;
$$


hence the equations for the $\lambda$ 's and $\lambda^{\prime \prime}$ s assume the form

$$
\begin{array}{r}
\left(\lambda_{1}^{\prime}-\eta_{1} \lambda_{m+2}\right) a_{i-1}+\left(\lambda_{2}^{\prime}-\lambda_{1}-\eta_{2} \lambda_{m+2}\right) a_{i}+\cdots+\left(\lambda_{m+2}^{\prime}-\lambda_{m+1}-\eta_{m+2} \lambda_{m+2}\right) a_{i+m}=0 \\
(i=1, \cdots, p-m-1) .
\end{array}
$$

The $m+2$ combinations of the $\lambda$ 's and $\lambda$ 's here involved, equated to zero, leave the $\lambda$ 's arbitrary, the $\lambda$ 's being expressed in terms of these. The $\rho$ 's consequently involve $m+2$ arbitrary parameters. The number of non-significant parameters being $m+1$, it is seen that one significant parameter appears in the terms of degree $p-m$ in the source, in consequence of the reduction from $m+3$ to $m+2$ of the number of derivates of degree $p-m-2$. If zero values be chosen for $m+1$ parameters, e. g., for $\lambda_{1}, \lambda_{2}, \ldots, \lambda_{1+m}$, the expressions for the $\rho$ 's become

$$
\rho_{i}=r_{i}+\lambda a_{i+m+1} \quad(i=0, \cdots, p-m),
$$

where $a_{p+1}$ is written for $\eta_{1} a_{p-m-1}+\eta_{2} a_{p-m}+\cdots$, and $\lambda$ for $\lambda_{m+2}$.

Now the relations connecting the $a$ 's, namely,

$$
\left\|\begin{array}{cccc}
a_{0} & a_{1} & \cdots & a_{p-m-2} \\
a_{1} & a_{2} & \cdots & a_{p-m-1} \\
a_{2} & & \\
\vdots & & & \\
a_{n+2} & a_{m+3} & \cdots & a_{p}
\end{array}\right\|=0,
$$

prove only that the highest terms can be eliminated from the derivates of degree $p-m-2$. If these same relations hold for the $b$ 's, $c$ 's, etc., then denoting the derivates by $D_{0}, D_{1}, \cdots, D_{m+2}$, we have

$$
D_{m+2}=\eta_{1} D_{0}+\eta_{2} D_{1}+\cdots+\eta_{m+2} D_{m+1},
$$

and the set of equations has become stationary. If however these relations do not hold for every set of coefficients $(b),(c), \ldots$, then for $D_{m+2}$ is substituted the equation of lower degree

$$
D_{m+2}-\left(\eta_{1} D_{0}+\eta_{2} D_{1}+\cdots \eta_{m+2} D_{m+1}\right)=0 \text {. }
$$

If the $b$ 's are not subject to the relations, the degree of this equation is $p-m-3$; if the $b$ 's are subject, but the $c$ 's not, the degree is $p-m-4$, and so on. Let this depressed equation be denoted by

$$
\left(A_{m+2}\right)^{p-m-2}+\left(B_{m+2}\right)^{p-m-3}+\cdots=0,
$$

and let this be inserted at the end of the derivates of degree $p-m-2$. The equations so far as now required are represented in the accompanying table, in which the degree of the terms in any column is given at the head of the column. 


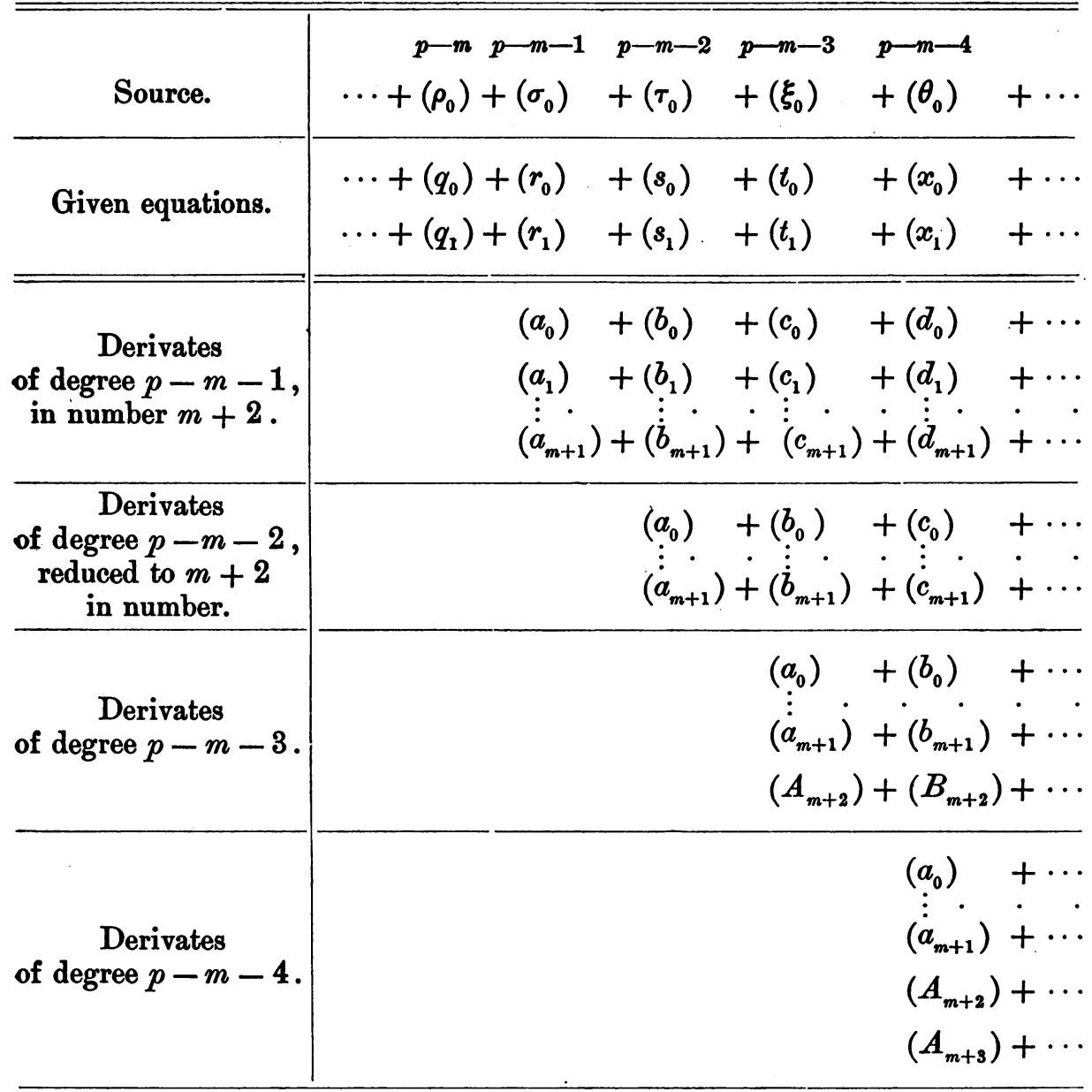

For the determination of the coefficients $\sigma$, which must involve in their expression $m+2$ non-significant parameters, we have the equations

$$
\begin{aligned}
& \sigma_{0}=s_{0}+\sum \lambda b_{0}+\kappa_{1} a_{0}+\cdots+\kappa_{m+2} a_{m+1}, \\
& \begin{aligned}
\sigma_{i}=s_{i}+\sum \lambda b_{i}+\kappa_{1} a_{i}+\cdots+\kappa_{m+2} a_{i+m+1} \\
\quad=s_{i}+\sum \lambda^{\prime} b_{i-1}+\kappa_{1}^{\prime} a_{i-1}+\cdots+\kappa_{m+2}^{\prime} a_{i+m} \quad(i=1, \cdots, p-m-2), \\
\quad=s_{p-m-1}+\sum \lambda^{\prime} b_{p-m-2}+\kappa_{1}^{\prime} a_{p-m-2}+\cdots+\kappa_{m+2}^{\prime} a_{p-1} .
\end{aligned} \\
& \begin{aligned}
\sigma_{p-m-1} \quad
\end{aligned}
\end{aligned}
$$

Now

$$
\begin{aligned}
\sum \lambda b_{1}-\sum \lambda^{\prime} b_{0} & =\lambda_{m+2} b_{m+2}-\left(\lambda_{1}^{\prime} b_{0}+\lambda_{2}^{\prime} b_{1}+\cdots+\lambda_{m+2}^{\prime} b_{m+1}\right) \\
& =\lambda\left\{b_{m+2}-\left(\eta_{1} b_{0}+\eta_{2} b_{1}+\cdots+\eta_{m+2} b_{m+1}\right)\right\} \\
& =\lambda A_{m+2} .
\end{aligned}
$$


Hence the equations for $\kappa, \kappa^{\prime}$ are

$$
\kappa_{1}^{\prime} a_{i-1}+\left(\kappa_{2}^{\prime}-\kappa_{1}\right) a_{i}+\cdots+\left(\kappa_{m+2}^{\prime}-\kappa_{m+1}\right) a_{i+m}-\kappa_{m+2} a_{i+m+1}=\lambda A_{i+m+1}
$$

that is,

$$
(i=1, \cdots, p-m-2) \text {, }
$$

$$
\begin{aligned}
&\left(\kappa_{1}^{\prime}-\eta_{1} \kappa_{m+2}\right) a_{i-1}+\left(\kappa_{2}^{\prime}-\kappa_{1}-\eta_{2} \kappa_{m+2}\right) a_{i} \\
&+\cdots+\left(\kappa_{m+2}^{\prime}-\kappa_{m+1}-\eta_{m+2} \kappa_{m+2}\right) a_{i+m}=\lambda A_{i+m+1} \\
&(i=1, \cdots, p-m-2) .
\end{aligned}
$$

Here every combination of $\kappa$ 's and $\kappa^{\prime}$ 's must be zero, as also the expressions on the right, on account of the number of the equations. Consequently unless every $A$ is zero, in which case the equation of depressed degree does not appear at this stage, we must have $\lambda=0$. Thus the significant parameter in the terms $\left(\rho_{0}\right)^{p-m}$ turns out to be deceptive; its value is now assigned, so that the $\rho$ 's are determinate, and the work proceeds as in the general case. The $m+2$ arbitrary parameters, $\kappa_{1}, \ldots, \kappa_{m+2}$, are all non-significant, and can conveniently be taken to be zero, and the $\sigma$ 's are consequently determinate.

If however every $A$ is zero, the equation substituted for $D_{m+3}$ takes its proper place among the derivates of degree $p-m-4$. (In the table just given the line $\left(A_{m+2}\right)^{p-m-3}+\left(B_{m+2}\right)^{p-m-4}+\cdots$ in the derivates of degree $p-m-3$ and the line $\left(A_{m+3}\right)^{p-m-4}+\cdots$ in those of degree $p-m-4$ are to be struck out.) The equations for $\tau$ are

$$
\begin{aligned}
& \tau_{0}=t_{0}+\sum \lambda c_{0}+\nu_{1} a_{0}+\cdots+\nu_{m+2} a_{m+1}, \\
& \tau_{i}=t_{i}+\sum \lambda c_{i}+\nu_{1} a_{i}+\cdots+\nu_{m+2} a_{i+m+1} \\
& =t_{i}+\sum \lambda^{\prime} c_{i-1}+\nu_{1}^{\prime} a_{i-1}+\cdots+\nu_{m+2}^{\prime} a_{i+m} \\
& (i=1, \cdots, p-m-3) \text {, } \\
& \tau_{p-m-2} \\
& =t_{p-m-2}+\sum \lambda^{\prime} c_{p-m-3}+\nu_{1}^{\prime} a_{p-m-3}+\cdots+\nu_{m+2}^{\prime} a_{p-2} \text {. }
\end{aligned}
$$

Here

$$
\begin{aligned}
\sum \lambda c_{1}-\sum \lambda^{\prime} c_{0} & =\lambda_{m+2} c_{m+2}-\left(\lambda_{1}^{\prime} c_{0}+\lambda_{2}^{\prime} c_{1}+\cdots+\lambda_{m+2}^{\prime} c_{m+1}\right) \\
& =\lambda\left\{c_{m+2}-\left(\eta_{1} c_{0}+\eta_{2} c_{1}+\cdots+\eta_{m+2} c_{m+1}\right)\right\} \\
& =\lambda A_{m+2} .
\end{aligned}
$$

Consequently the $\nu$ 's are determined by the set of equations

$$
\begin{array}{r}
\nu_{1}^{\prime} a_{i-1}+\left(\nu_{2}^{\prime}-\nu_{1}\right) a_{i}+\cdots+\left(\nu_{m+2}^{\prime}-\nu_{m+1}\right) a_{i+m}-\nu_{m+2} a_{i+m+1}=\lambda A_{i+m+1} \\
(i=1, \cdots, p-m-3),
\end{array}
$$

that is, by the set of equations

$$
\begin{array}{r}
\left(\nu_{1}^{\prime}-\eta_{1} \nu_{m+2}\right) a_{i-1}+\left(\nu_{2}^{\prime}-\nu_{1}-\eta_{2} \nu_{m+2}\right) a_{i}+\cdots=\lambda A_{i+m+1} \\
(i=1, \cdots, p-m-3) .
\end{array}
$$


Precisely as before, unless every $A=0$, it follows that $\lambda=0$; the parameter $\lambda$ is only temporary. The $\tau$ 's contain only non-significant parameters in any case, and the work proceeds exactly as before, the first derivates whose multipliers are not zero being those of the degree where the dropped one reappears. The partial agreement of derivates may occur again and again, but this causes no complication; each temporary parameter which presents itself when the law of unit increase is interrupted bas its value determined when this law again comes into operation. Thus we have the result that partial agreement of the derivates of any degree produces no effect on the parameters in the source, while absolute agreement, the equations having become a stationary set, is expressed by the occurrence of a single parameter in the terms of degree $p-m$, where the natural derivates of degree $p-m-2$ are subject to a relation by which their number is diminished by unity.

18. Stationary set.-The equations for this case are the same as those of the table given above, with the omission of the lines

$$
\left(A_{m+2}\right)^{p-m-3}+\cdots, \quad\left(A_{m+2}\right)^{p-m-4}+\cdots, \cdot\left(A_{m+3}\right)^{p-m-4}+\cdots,
$$

etc. The equations for $\sigma$, already given, lead to the equations for $\kappa, \kappa^{\prime}$, these giving every $\kappa^{\prime}$ in terms of $\kappa^{\prime}$ 's, so that there are $\dot{m}+2$ parameters, $\kappa_{1}, \cdots, \kappa_{m+2}$, all non-significant. Hence the $\sigma$ 's are determinate. In like manner the $\tau$ 's, and all succeeding sets of coefficients, are determinate; the source involves precisely the one parameter $\lambda$ before we arrive at the terms of degree $k$. Since we are dealing with equations which have become stationary, there are $k$ equations of degree $k$, by means of which the terms of degree $k$ in the source can be reduced to $A_{2} z_{0}^{k}$. The source is therefore

$$
E^{p}+A_{1} E^{q}+A_{2} z_{0}^{k}=0
$$

an equation which involves two parameters, as before.

19. One case remains for investigation, that in which the two given equations yield only two derivates of the next lower degree. This requires that

$$
a_{i+1}=\eta_{1} a_{i-1}+\eta_{2} a_{i} \quad(i=1, \cdots, p-1) .
$$

It has been shown ( $\$ 14)$ that if $a_{p+1}$ be written for $\eta_{1} a_{p-1}+\eta_{2} a_{p}$, the terms $\left(a_{0}\right)^{p}$ in the source are $\lambda_{1}\left(a_{0}\right)^{p}+\lambda_{2}\left(a_{1}\right)^{p}$. Hence

where

$$
D_{x} E=\lambda_{1} E_{1}+\lambda_{2} E_{2}, \quad D_{y} E=\lambda_{1} E_{2}+\lambda_{2} E_{3},
$$

Consequently

$$
E_{3}=\left(a_{2}\right)^{p}+\cdots=\eta_{1} E_{1}+\eta_{2} E_{2} \text {. }
$$

$$
D_{y} E=\lambda_{2} \eta_{1} E_{1}+\left(\lambda_{1}+\lambda_{2} \eta_{2}\right) E_{2}=\lambda_{1}^{\prime} E_{1}+\lambda_{2}^{\prime} E_{2} .
$$


The equations for $\beta$ are therefore

$$
\begin{aligned}
\beta_{0}=\lambda_{1} b_{0}+\lambda_{2} b_{1}+\kappa_{1} a_{0}+\kappa_{2} a_{1}, & \\
\beta_{i}=\lambda_{1} b_{i}+\lambda_{2} b_{i+1}+\kappa_{1} a_{i}+\kappa_{2} a_{i+1} & =\lambda_{1}^{\prime} b_{i-1}+\lambda_{2}^{\prime} b_{i}+\kappa_{1}^{\prime} a_{i-1}+\kappa_{2}^{\prime} a_{i} \\
& =\lambda_{1}^{\prime} b_{p-2}+\lambda_{2}^{\prime} b_{p-1}+\kappa_{1}^{\prime} a_{p-2}+\kappa_{2}^{\prime} a_{p-1} .
\end{aligned}
$$

Hence

$$
\begin{aligned}
\kappa_{1}^{\prime} a_{i-1}+\left(\kappa_{2}^{\prime}-\kappa_{1}\right) a_{i}-\kappa_{2} a_{i+1}= & \lambda_{1} b_{i}+\lambda_{2} b_{i+1}-\lambda_{2} \eta_{1} b_{i-1}-\left(\lambda_{1}+\lambda_{2} \eta_{2}\right) b_{i} \\
& =\lambda_{2}\left(b_{i+1}-\eta_{1} b_{i-1}-\eta_{2} b_{i}\right) \quad(i=1, \cdots, p-2) .
\end{aligned}
$$

Since $a_{i+1}=\eta_{1} a_{i-1}+\eta_{2} a_{i}$, the equations become, on writing

$$
\begin{aligned}
& A_{i+1}=b_{i+1}-\eta_{1} b_{i-1}-\eta_{2} b_{i}, \\
& \left(\kappa_{1}^{\prime}-\eta_{1} \kappa_{2}\right) a_{i-1}+\left(\kappa_{2}^{\prime}-\kappa_{1}-\eta_{2} \kappa_{2}\right) a_{i}=\lambda_{2} A_{i+1} \quad(i=1, \cdots, p-2) .
\end{aligned}
$$

Consequently unless every $A=0$, that is, unless the $b$ 's are subject to the same relation as the $a$ 's, we have $\lambda_{2}=0$. That is to say, if the three derivates of the given equations are equivalent to two of degree $p-2$ and one of the next lower degree, the terms of degree $p$ in the source reduce to $\left(a_{0}\right)^{p}$. If however the $b$ 's are subject to this relation, we have $A=0$. In either case, $\kappa_{1}^{\prime}$ and $\kappa_{2}^{\prime}$ are expressed in terms of $\kappa_{1}$ and $\kappa_{2}$; the $\beta$ 's involve two parameters, both non-significant, and therefore zero if we choose. Precisely the same argument applies to the following sets of terms; and we obtain the result that if

$$
D_{y} E_{2}-\eta_{1} D_{x} E_{1}-\eta_{2} D_{y} E_{1}
$$

is of degree lower than $p-2$, the parameter in the terms $\left(a_{0}\right)^{p}$ has its value determined ultimately, so that the source is not affected by this partial agreement; while if this combination of the derivates vanishes, so that the two given equations yield precisely two derivates (as a necessary consequence of which there are only two derivates of any degree), the source is

$$
\lambda_{1}\left\{\left(a_{0}\right)^{p}+\cdots\right\}+\lambda_{2}\left\{\left(a_{1}\right)^{p}+\cdots\right\}+\text { terms in }\left(z_{0}^{2}, z_{1}^{2}, z_{2}^{2}\right)=0 .
$$

Since there are two equations of degree two, the ratios $z_{0}^{2}: z_{1}^{2}: z_{2}^{2}$ are known; consequently the source is

$$
\lambda_{1}\left\{\left(a_{0}\right)^{p}+\cdots\right\}+\lambda_{2}\left\{\left(a_{1}\right)^{p}+\cdots\right\}+\lambda_{3} z_{0}^{2}=0,
$$

and the two parameters are present as in the other cases.

20. The results obtained are that the general source of degree $p$ involves in its equation two arbitrary parameters. The source can be written as

where

$$
A_{0} E^{p}+A_{1} E^{q}+A_{2} E^{k}=0,
$$
(i) $q=k$,
(ii) $p>q>k$,
(iii) $q=p, k=2$. 
If now the source is to relate to two given curves, $u, v$, for which the two given equations and all their derivates are satisfied, this supplies two equations for the determination of $A_{0}: A_{1}: A_{2}$. It is conceivable that for special curves there may not be a unique determination of these parameters; but as regards cases (i) and (ii) this cannot happen unless the curves are chosen so as to satisfy an equation of degree $\leqq p-1$, not included among those given. For in case (i), if there is still an undetermined parameter, the source

becomes

$$
A_{0} E^{p}+A_{1} E_{1}^{k}+A_{2} E_{2}^{k}=0
$$

$$
A_{0} E^{p}+B E^{k}=0,
$$

showing that there is an additional equation of degree $k, E^{k}=0$, not obtainable from the derivates of the two given equations. Similarly in case (ii) there is an additional equation of degree $q \leqq p-1$, which is not included among those given, though its derivates are included. For the arbitrary parameter which presents itself in the determination of the coefficients $\rho$ is the multiplier of an expression

$$
a_{m+1} z_{0}^{p-m}+a_{m+2} z_{1}^{p-m}+\cdots+a_{p+1} z_{p-m}^{p-m}+\cdots,
$$

whose two derivates are $\left(a_{m+1}\right)^{p-m-1}+\cdots$, and $\left(a_{m+2}\right)^{p-m-1}+\cdots$ Of these, the first is the last of the $m+2$ derivates of degree $p-m-1$, while in virtue of the relations connecting the $a$ 's, the second is linearly expressible in terms of the last two of these $m+2$ derivates. Hence all the derivates of $A E^{p}+B E^{q}=0$ are members of the given system. This new equation is of degree $q=p-m$, while the reduction in the number of derivates occurs at degree $q-2$. As the reduced number is $k$, there are $k$ derivates of degree $q-1$; beginning with these, the set of $k$ equations is stationary. Now a stationary set may be derived from $k-1$ equations; but it may be derived from $k$ equations, in which case the set has become stationary one stage earlier. Thus such a set of equations as that indicated in the diagram by the $* * *$ may have to be completed, for particular curves, by the equations $O \circ \circ$, and again by $\square \square \square$; but these do not arise from the two given equations.

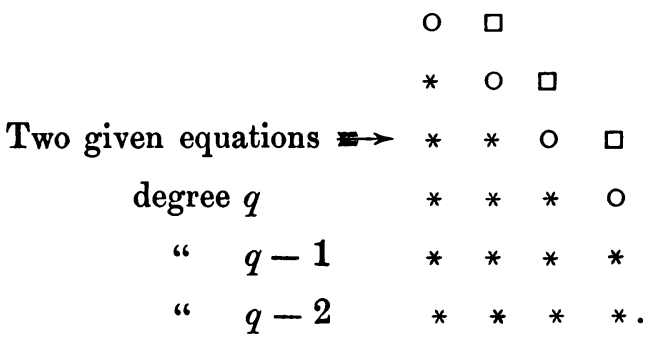


In both these cases there is strictly only one source of degree $p$, arising from the given equations. But case (iii) is different. The source is now

$$
A_{0} E_{1}^{p}+A_{1} E_{2}^{p}+A_{2} E^{2}=0 .
$$

If the two given curves fail to determine the parameters uniquely, there are two distinct sources of degree $p$. The explanation is simple enough. The set is stationary, including the two given equations. It may arise from one equation; but it may also arise from two equations, just as a stationary set of $k$ equations may arise from $k-1$ equations or from $k$ equations.

21. In a similar manner can be proved the generalized theorem of ascent:

If $m$ equations of degree $p$ yield not more than $m+1$ derivates of degree $p-1$, they arise from not less than $m-1$ equations of degree $p+1$.

Comparing the two derivates of an assumed source

$$
\left(a_{0}\right)^{p+1}+\left(\beta_{0}\right)^{p}+\left(\gamma_{0}\right)^{p-1}+\cdots=0
$$

with general linear functions of the given equations, and modifying the resulting equations by means of relations arising from the fact that the $2 m$ derivates of the given equations reduce to $m+1$, we find that the $a$ 's involve $m-1$ arbitrary parameters. Assigning any values to these we find that the source is determinate when the values of two parameters in later terms are obtained by means of two given curves. The theorem follows also from the application about to be made of the simple theorem of ascent.

22. Proof of Theorem (1).-By means of the theorem of ascent, it can be shown that any two curves determine a one-set system of equations. For this we start with the equations of degree $k$ and $k+1$, whose numbers are known to be
(i) $k-1$ and $k-2$,
(ii) $k$ and $k-1$,
(iii) $k$ and $k$.

The equations of degree $k+1$ have those of degree $k$ for derivates; hence one of two cases must occur. Either (1) they can be written so that any two successive ones have a common derivate, or (2) they fall into distinct sets, each set giving rise to a part of the derivates of degree $k$. This last case will be considered separately, and then it will be shown that it does not differ essentially from the first case.

In the first case, by hypothesis, the equations can be written so as to have common derivates; we can therefore ascend step by step, obtaining in general one equation fewer of the next rank, and so on. In (iii) the whole set is sta- 
tionary to begin with; the theorem of ascent, applied to the equations in pairs, shows at once that the number of equations of degree $k+2$ will be either $k-1$ or $k$.

In every event, having arrived at $h$ equations of degree $q$, we can ascend to their sources of degree $q+1$, obtaining either $h$ or $h-1$ of these. Hence one of two results is inevitable; we arrive finally at a single equation, or else we arrive at a minimum number, $h$, from which we can ascend indefinitely, obtaining $h$ equations of every higher degree. This however is impossible, for it is shown in $\S 28$ that the number of points of intersection of two curves, falling at the origin, is at least as great as* the number of the equations due to their behavior at the origin.

No equations can exist except those here determined; for since their derivates must be satisfied, those of degree $k+1$ and $k$ are included among the equations used as a starting point for the process of ascent; and the investigations comprised in the proof of the theorem of ascent show that we have obtained every possible source.

23. It has now to be shown that case (2), when the equations break up into sets, does not differ materially from case (1). Suppose, for the sake of generality, that this occurs at degree $q$; that is, it is to be supposed that $m_{1}$ equations of degree $q$ give $m_{1}^{\prime}$ of degree $q-1$ as derivates, while the remaining $m_{2}$ of degree $q$ give the remaining $m_{2}^{\prime}$ of degree $q-1$. If this can happen, the process of ascent applied to each set separately proves the existence of a prime equation for each set. Now if the derivates of degree $k$, of these two sets, were not entirely independent, the ordinary process of ascent would apply, leading ultimately to one source. Hence the only case to be considered is that where the separation makes itself felt at the very outset, in the equations of degrees $k$ and $k+1$. It has been shown that the number of equations of degree $k+1$ cannot fall short of the number of degree $k$ by more than unity; hence one at least of the two sets into which the equations break up must be stationary. All that has to be proved, in order to establish the applicability of the process of the last section, is that the equations belonging to the two separate sets do not form the complete system, that additional equations result from the combination of the two sets.

Of the equations of degree $k$, in number $k-1$, let $m$ result from the stationary set, $q$ from the progressive set. Let the number of equations in the stationary set, obtained from this alone, be $m$ at degree $p, m-1$ at degree

\footnotetext{
* As shown in $\$ 29$, these numbers are as a matter of fact equal; but the proof of their equality makes use of the result of the present section, while the proof of the property here stated does not.
} 
$p+1$. There may be equations of the remaining set at this degree (fig. 1), or there may not (fig. 2).

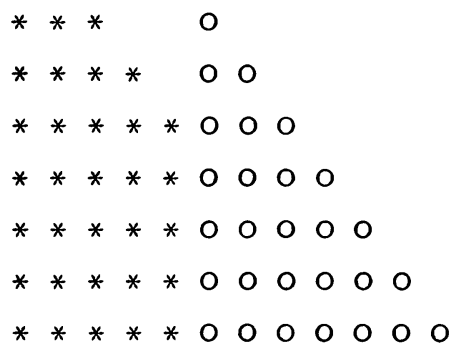

FIG. 1.

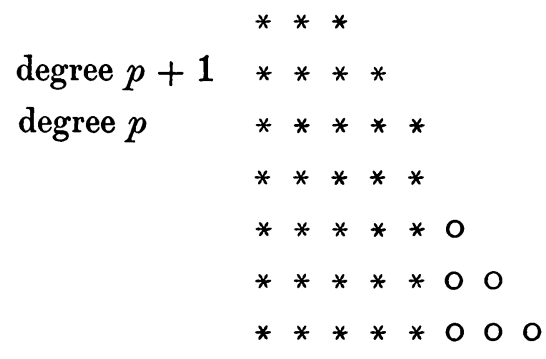

Fia. 2.

It is sufficient to prove that there exists another equation of degree $p+1$, not resulting from either set alone.

If the source of the stationary set be written

$$
\left(a_{0}\right)^{P}+\left(b_{0}\right)^{P-1}+\left(c_{0}\right)^{P-2}+\cdots=0,
$$

the $m-1$ equations of degree $p+1$ are obtained in the form

$$
\left(a_{i}\right)^{p+1}+\left(b_{i}\right)^{p}+\left(c_{i}\right)^{p-1}+\cdots+\left(h_{i}\right)^{k+2}+\left(j_{i}\right)^{k+1}+\left(k_{i}\right)^{k}=0 \quad(i=0, \cdots, m-2) .
$$

The reduction of the $m+1$ derivates of the degree $p-1$ to $m$ depends on the vanishing of the determinants

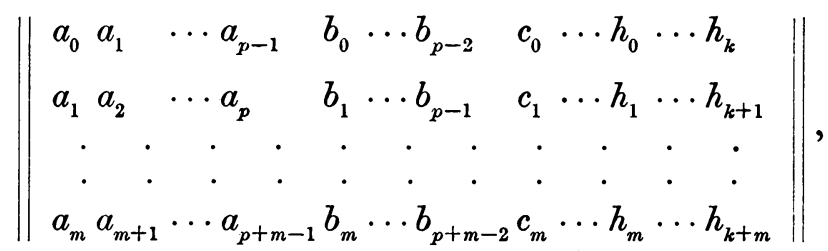

or, in the form here required, on the existence of linear relations

$$
\begin{gathered}
a_{m}=\eta_{1} a_{0}+\eta_{2} a_{1}+\eta_{3} a_{2}+\cdots+\eta_{m} a_{m-1}, \\
a_{m+1}=\eta_{1} a_{1}+\eta_{2} a_{2}+\eta_{3} a_{3}+\cdots+\eta_{m} a_{m}, \\
\cdot \cdot \cdot \cdot \cdot \cdot \cdot \cdot \cdot \cdot \cdot \cdot \cdot \cdot \cdot \cdot \cdot \cdot \cdot \cdot \cdot \cdot \cdot \eta_{m} \cdot h_{k+m} .
\end{gathered}
$$

It is to be noted that $a$ 's, $b$ 's, $\ldots, h$ 's can be built up with any suffix by this law of formation.

The proof of the existence of another equation of degree $p+1$ is most easily followed by reference to the accompanying table, in which the accented letters refer to the progressive set, whose source is

$$
\left(a_{0}^{\prime}\right)^{P^{\prime}}+\left(b_{0}^{\prime}\right)^{P^{\prime}-1}+\cdots=0 .
$$




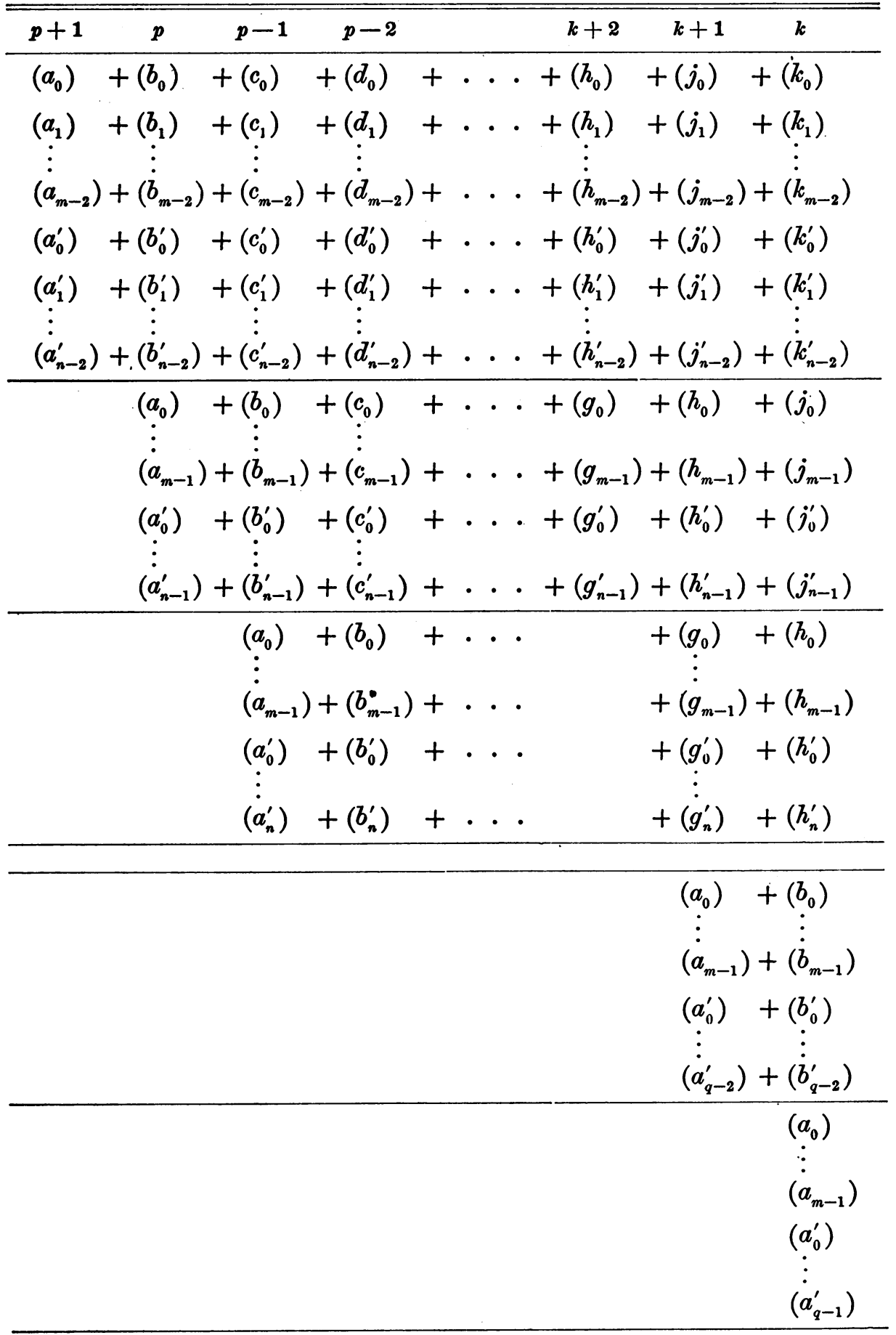


If the general equation of degree $p+1$ be written in the form

$$
(a)^{p+1}+(\beta)^{p}+(\gamma)^{p-1}+\cdots \text { etc. }=0,
$$

comparison of its derivates with linear functions of the given equations, carried so far as to include the terms of degree $k+1$ in these, at once suggests the possibility of a source $S=0$, in addition to those given in the table, where

$$
S=\left(a_{m-1}\right)^{p+1}+\left(b_{m-1}\right)^{p}+\cdots+\left(h_{m-1}\right)^{k+2}+\left(J_{m-1}\right)^{k+1}+\left(K_{m-1}\right)^{k} .
$$

The $J$ 's in this cannot be put equal to the $j$ 's of the given equation; if they could, the source would be derived from the stationary set alone, contrary to the hypothesis that there are only $m-1$ equations of degree $p+1$ belonging to this set. (The impossibility of determining the $J$ 's in this manner arises algebraically from the fact that the $j$ 's are not subject to the linear relations that prevail for the $a$ 's, $b$ 's, etc.)

Since the two derivates of $S$ must be expressible in terms of the given equations of degree $p$ and their derivates, it is at once evident that $D_{x} S$ must agree with $E_{m-1}^{p}$, and that

must agree with

$$
\begin{gathered}
D_{y} S \quad\left[=\left(a_{m}\right)^{p}+\left(b_{m}\right)^{p-1}+\cdots\right] \\
\eta_{1} E_{0}^{p}+\eta_{2} E_{1}^{p}+\cdots+\eta_{m} E_{m-1}^{p},
\end{gathered}
$$

each modified by linear multiples of the equations of degree $k$. Let $J^{\prime}$ be written for $\eta_{1} j_{0}+\eta_{2} j_{1}+\cdots+\eta_{m} j_{m-1}$, etc., then the equations to be satisfied are those derived from

These are

$$
\begin{aligned}
& \left(J_{m-1}\right)^{k} \equiv\left(j_{m-1}\right)^{k}+\sum \lambda\left(a_{0}\right)^{k}+\sum \lambda^{\prime}\left(a_{0}^{\prime}\right)^{k}, \\
& \left(J_{m}\right)^{k} \equiv\left(J_{m}^{\prime}\right)^{k}+\sum \mu\left(a_{0}\right)^{k}+\sum \mu^{\prime}\left(a_{0}^{\prime}\right)^{k} .
\end{aligned}
$$

$$
\begin{aligned}
& J_{m-1}=j_{m-1}+\sum \lambda_{1} a_{0}+\sum \lambda_{1}^{\prime} a_{0}^{\prime}, \\
& J_{m} \quad=j_{m}+\sum \lambda_{1} a_{1}+\sum \lambda_{1}^{\prime} a_{2}=J_{m}^{\prime}+\sum \mu_{1} a_{0}+\sum \mu_{1}^{\prime} a_{0}^{\prime}, \\
& \text { - . } \\
& J_{m-1+k}=j_{m-1+k}+\sum \lambda_{1} a_{k}+\sum \lambda_{1}^{\prime} a_{k}^{\prime}=J_{m-1+k}^{\prime}+\sum \mu_{1} a_{k-1}+\sum \mu_{1}^{\prime} a_{k-1}^{\prime} \text {, } \\
& J_{m+k} \quad=J_{m+k}^{\prime}+\sum \mu_{1} a_{k}+\sum \mu_{1}^{\prime} a_{k}^{\prime}
\end{aligned}
$$

Here there are $m \lambda$ 's and $\mu$ 's, and $q \lambda$ 's and $\mu$ 's, where $m+q=k-1$. The double values for $J_{m}, \cdots, J_{m-1+k}$ yield $k$ equations of the type

$$
\begin{aligned}
& \left(\mu_{1}-\eta_{1} \lambda_{m}\right) a_{0}+\left(\mu_{2}-\lambda_{1}-\eta_{2} \lambda_{m}\right) a_{1}+\cdots+\left(\mu_{m}-\lambda_{m-1}-\eta_{m} \lambda_{m}\right) a_{m-1} \\
& +\mu_{1}^{\prime} a_{0}^{\prime}+\left(\mu_{2}^{\prime}-\lambda_{1}^{\prime}\right) a_{1}^{\prime}+\cdots+\left(\mu_{q}^{\prime}-\lambda_{q-1}^{\prime}\right) a_{q-1}^{\prime}-\lambda_{q}^{\prime} a_{q}^{\prime}+J_{m}^{\prime}-j_{m}=0 .
\end{aligned}
$$


In these there are $m+q+1(=k)$ combinations of the unknown quantities; and since the absolute terms in these $k$ equations are not zero we obtain for each of these combinations a determinate value, in general different from zero. These leave the $m . \lambda$ 's arbitrary, as also $\lambda_{1}^{\prime}, \lambda_{2}^{\prime}, \ldots, \lambda_{q-1}^{\prime}$, the $m \mu$ 's and $\mu_{2}^{\prime}, \mu_{3}^{\prime}, \ldots, \mu_{q}^{\prime}$ being expressed in terms of these; but they assign to $\lambda_{q}^{\prime}$ and $\mu_{1}^{\prime}$ definite numerical values, which it will be shown immediately are in general different from zero. The $m \lambda$ 's and $q-1 \lambda^{\prime \prime}$ s appearing in the general values for $\left(J_{m-1}\right)^{k+1}$ are non-significant; they simply allow for the modification of the source by multiples of the equations of degree $k+1$; if these be taken to be zero, and $L$, $M$ be written for $\lambda_{q}^{\prime}, \mu_{1}^{\prime}$, the $J$ 's become

$$
\begin{aligned}
& J_{m-1}=j_{m-1}+L a_{q-1}^{\prime}, \\
& \begin{aligned}
J_{m+i} & =j_{m+i}+L a_{q+i}^{\prime}
\end{aligned}=J_{m+i}^{\prime}+M a_{i} \quad(i=0, \cdots, k-1), \\
& J_{m+k} \quad=J_{m+l k}^{\prime}+M a_{k}^{\prime} .
\end{aligned}
$$

Hence the source is

$$
S=\left(a_{m-1}\right)^{p+1}+\left(b_{m-1}\right)^{p}+\cdots+\left(h_{m-1}\right)^{k+2}+\left(j_{m-1}\right)^{k+1}+L\left(a_{q-1}^{\prime}\right)^{k+1}+(K)^{k},
$$

where the coefficients in $(K)^{k}$, equivalent to two independent ones, are to be determined by means of the two given curves. (The $j_{m+l}$, etc., denote definite numerical quantities which present themselves in the solution of the equations in such a manner that these names are the obvious ones for them.) The component $L\left(a_{q-1}^{\prime}\right)^{k+1}$ in this source indicates that it is derived from the two sets together; the vanishing of $L$ would mean that the source belongs to the one set, which is contrary to the hypothesis. Thus it is seen that the separation of the equations into sets at the foundation does not indicate any permunent cleavage; the sets are connected by this equation of degree $p+1$. It may be noted that

$$
D_{x} S=D_{y}\left(E_{m-2}^{p+1}+L{E_{q-2}^{\prime k+1}}^{\prime k}\right)
$$

where $E^{\prime}$ indicates the equation $\left(a_{q-2}^{\prime}\right)^{k+1}+\left(b_{q-2}^{\prime}\right)^{k}=0$ of the second set. This shows that we are in a position to continue the process of ascent.

While this is all that is necessary for the proof of the applicability of the theorem of ascent, the form of the complete result is of interest. I have not worked it out to the end, but a few steps make the general law perfectly plain. Let $E_{h}^{p+t}$ be written for $\left(a_{h}\right)^{p+t}+\left(b_{h}\right)^{p+t-1}+\cdots+\left(j_{h}\right)^{k+t}$; the additional equation of degree $p+1$ has been shown to differ from $E_{m-1}^{p+1}$ at the terms of degree $k+1$ by a multiple of $E_{q-1}^{\prime k+1}$; there are two extra equations of degree $p+2$ which differ from $E_{m-2}^{p+2}$ and $E_{m-1}^{p+2}$ at the terms of degree $k+2$ by multiples of $E_{q-2}^{\prime k+2}, E_{q-1}^{\prime k+2}$ and expressions of lower degree; three extra equations of degree $p+3$ which differ from $E_{m-3}^{p+3}, E_{m-2}^{p+3}$ and $E_{m-1}^{p+3}$ by multiples of $E_{q-3}^{\prime^{k+3}},{E_{q-2}^{\prime k+3}}^{\prime 2}$, $E_{q-1}^{\prime k+3}$ and lower expressions, and so on, until finally the source of the second 
set, $E_{0}^{\prime q+k}$, makes its appearance. There are still $m$ equations of the next higher degree, from whose $m+1$ derivates the source ${E_{0}^{\prime q+k}}^{q+k}$ results by elimination of the highest terms, namely, those with coefficients $a$ to $h$ included. After this, the equations diminish in number by unity at every stage. (See $\S 10$.)

The very slight modifications required when the second set becomes stationary at or before the degree $k$, in which case $m+q$ is equal to $k$ instead of $k-1$, do not interfere with this conclusion; nor does the breaking up of the stationary set further - the different sets can be compounded in turn.

24. It has now been shown that the equations obtained by the process of ascent do not break away into sets; they form one system, and lead to one equation of some finite degree $p$, which is the prime equation for the base-point determined by the two curves. As all the equations due to the nature of the curves at the origin are included in the system, it follows that the base-point determined by the two curves is a one-set point. We have now proved the

THEOREM : two curves satisfy precisely one prime equation, which is the first theorem of $\S(2)$.

If two or more sources are given for two curves, then by means of this theorem it is seen that they and their derivates can be exhibited as component parts of a more extensive system, arising from the one prime equation that is satisfied by the two curves; and if an expression $E$ and all its derivates vanish, then $E$ is either the prime equation itself, or one of its derivates. The system derived from $E$ is either a part or the whole of the one-set system.

25. Proof. of Theorem (2).-The results already obtained enable us to find the equations satisfied by more than two curves. Suppose that $m+1$ equations of degree $p$ have been found satisfied by $h+2$ curves, and that the process of ascent for two of the curves yields $m$ equations of degree $p$; the complete system satisfied by the two is

$$
\lambda_{1} E_{1}+\lambda_{2} E_{2}+\cdots+\lambda_{m} E_{m}=0 .
$$

Imposing on the $\lambda$ 's the conditions afforded by the remaining $h$ curves, namely,

$$
\lambda_{1} E_{1}^{(i)}+\lambda_{2} E_{2}^{(i)}+\cdots+\lambda_{m} E_{m}^{(i)}=0 \quad(i=1, \cdots, h) .
$$

we shall obtain the system satisfied by the $h+2$ curves. If $h<m$, this is

$$
\begin{aligned}
& \begin{array}{lllll}
E_{1} & E_{2} & \cdots & E_{h} & \lambda_{h+1} E_{h+1}+\cdots+\lambda_{m} E_{m}
\end{array} \\
& \begin{array}{ccccc}
E_{1}^{(1)} & E_{2}^{(1)} & \cdots & E_{h}^{(1)} & \lambda_{h+1} E_{h+1}^{(1)}+\cdots+\lambda_{m} E_{m}^{(1)} \\
. & . & . & . & .
\end{array} \\
& E_{1}^{(h)} \quad E_{2}^{(h)} \ldots E_{h}^{(h)} \lambda_{h+1} E_{h+1}^{(h)}+\cdots+\lambda_{m} E_{m}^{(h)}
\end{aligned}
$$


Hence if $m>h$, there are $m-h$ independent equations of degree $p+1$; that is, if the number of equations is not less than the number of curves, we can ascend to a smaller number.

But this smaller number will not suffice unless its derivates, whose number cannot exceed $2(m-h)$, yield all the $m+1$ equations. We must have therefore;

$$
2(m-h) \geqq m+1, \quad \text { that is, } m-h \geqq h+1 .
$$

The test is, therefore, that the number of equations of degree $p+1$ shall be not less than $h+1$.

If the equations of degree $p+1$ are not so many as $m+1$, they do not form the complete system, and a certain number of those of degree $p$ must be given independently. Inasmuch as there are at most $2(m-h)$ equations of degree $p$ derived from those of degree $p+1$, there must be given independently at least $m+1-2(m-h)$ of degree $p$; hence the system of equations contains at least $m-h+m+1-2(m-h)$, that is, $h+1$. Thus with $h+2$ curves to attend to, we can ascend to $h+1$ equations, but no further; and we have the

Theorem: $t+1$ independent curves satisfy precisely $t$ prime equations, which is the second theorem of $\S 2$.

To find the equations for given curves, the process of ascent seems simplest. It is most conveniently applied to two of the curves in the first place, after which linear functions of the equations obtained must be chosen so as to be satisfied by the remaining curves.

\section{The number of intersections of two curves at the origin.}

26. The proof that the number of points of intersections of two curves at a common point is the same as the number of equations contained in the one-set system proper to the point depends on a particular arrangement of the equations. It must be noticed in the first place that in the prime equation no coefficients $z_{h}^{p}$ of the highest degree need be considered to be absent, for this can be obviated by a change of axes if necessary.

In the diagram in which the $x$-derivates are arranged in vertical lines, namely,

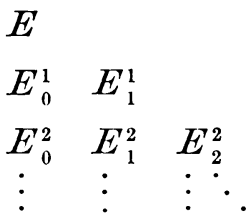

let $E_{1}^{1}$ be replaced by $E_{1}^{1}+M_{1} E_{0}^{1}+M_{2} E_{0}^{2}+\cdots$, the multipliers $M$ being chosen so as to remove every $z_{0}$; thus $E_{1}^{1}$ as modified contains terms

$$
\left(z_{1}^{p-1}+\cdots\right)+\left(z_{1}^{p-2}+\cdots\right)+\cdots
$$


When all the members of the column are treated in a similar manner, the same modification is produced; moreover, the relation of each member to the one above remains unaltered, it is still the $x$-derivate. Similarly, let $E_{2}^{2}$ be replaced by an expression containing no terms $z_{0}, z_{1}$, and so on. If it should happen that not only every $z_{0}$, but also every $z_{1}$, disappears from $E_{1}^{1}$, this must be taken as the head of the third column, and its $y$-derivate as head of the second column, with similar modifications in the arrangement if other $z$ 's disappear. If every $z^{h}$ disappears when any $E$ of degree $h$ is modified with a view to eliminating a particular $z^{h}$, the resulting equation is of lower degree, but this does not affect the arrangement of columns. Since the equations of degree $k-1$ reduce to

$$
z_{0}^{k-1}=0, z_{1}^{k-1}=0, \cdots, z_{k-1}^{k-1}=0,
$$

it follows that every one of the first $k$ suffixes is represented by a column; the diagram now consists of $k$ columns, not necessarily arranged according to height, whose bases are the equations

$$
\left(z_{0}\right)^{k-1}=0,\left(z_{1}\right)^{k-1}=0,\left(z_{2}\right)^{k-1}=0, \cdots,\left(z_{k-1}\right)^{k-1}=0,
$$

where $\left(z_{h}\right)^{k-1}$ is written for a linear function of $z^{k-1}$ 's, with no suffix below $h$. There are also to be taken into account the equations expressing that every $z$ with an index $<k-1$ vanishes; when these are arranged below the others, according to their suffixes, the diagram represents all the equations belonging to the base-point. The numbers of equations in the columns are

$$
p_{0}+1, p_{1}, p_{2}-1, \cdots, p_{s}-(s-1), \cdots p_{k-1}-(k-2),
$$

where the degree of the leading equation of any column is denoted by $p$ with the proper suffix.

Figs. (1) and (2) illustrate possible forms with $p_{0}=10, k=5$.

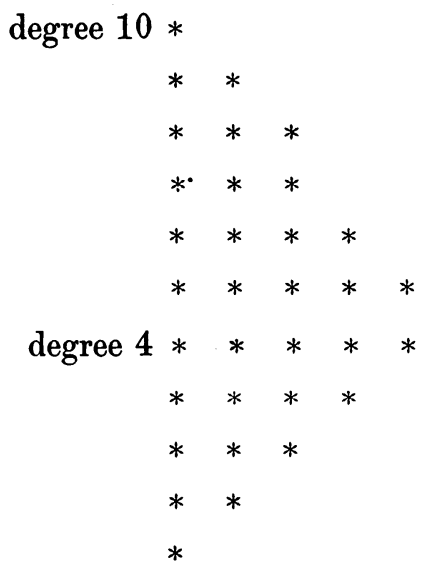

FIG. 1. degree $10 *$

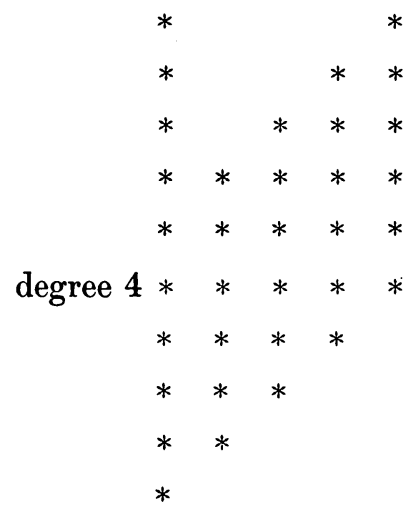

FIG. 2. 
27. If an equation and all its derivates are satisfied, it is known that the equation is a member of the one-set system. For the recognition of this, however, it is not necessary that the fact be stated for all the derivates. If an expression $E$ and its first $k y$-derivates $E_{1}^{1}, E_{2}^{2}, \cdots, E_{k}^{k}$, as well as all the $x$ derivates of these, vanish for two curves that have at the origin a multiple point of order $k$, then all the derivates of $E$ vanish, so that $E$ is a member of the one-set system proper to the point. The proof of this depends on showing that the equations are not independent. If at every degree the $x$-derivates of the $k+1$ equations $E, E_{1}^{1}, E_{2}^{2}, \cdots, E_{k}^{k}$ are independent, they lead to $k+1$ equations of degree $k$, which determine the order of the multiple point as $k+1$ instead of $k$. Hence the $x$-derivates are not independent at every degree. But if a linear relation connects the $x$-derivates of degree $h$, and the equations of degree $h+1$ are combined as indicated by this same linear relation, the only $z$ 's remaining will be $z_{h+1}^{h+1}, z_{h}^{h}, z_{h-1}^{h-1}$, etc. This gives a relation in coefficients of powers of $y$ only, which can be avoided by taking axes with no specialized relation to the curve. Hence we see that the $k+1$ equations of degree $p$, where $p$ is equal to the degree of $E_{k}^{k}$, cannot be independent; they must reduce to $k$ by means of a linear relation. If now this relation does not involve $E_{k}^{k}$, let it involve no $E^{k}$ beyond $E_{h}^{k}$, then

$$
E_{h}^{k}=\left(E_{0}^{k}, E_{1}^{k}, \cdots, E_{h-1}^{k}\right)^{1} .
$$

But since $E_{h}^{k}=D_{x} E_{h}^{k-1}$, this may be written

$$
D_{x} E_{h}^{k-1}=D_{x}\left(E_{0}^{k-1}, E_{1}^{k-1}, \cdots, E_{h-1}^{k-1}\right)^{1} ;
$$

this shows that from $E_{0}^{k-1}, E_{1}^{k-1}, \ldots, E_{h}^{k-1}$ all $z$ 's except $z_{p+1}^{p+1}, z_{p}^{p}, \ldots$, etc. can be eliminated, a particular case which as we have seen can be avoided. Consequently the relation connecting the derivates of degree $p$ does involve $E_{l k}^{k}$; it gives therefore

Hence

$$
E_{k}^{k}=\text { a linear function of } E_{0}^{k}, \cdots, E_{k-1}^{k} \text {. }
$$

$$
\begin{aligned}
D_{y} E_{k}^{k} & =\text { a linear function of the } y \text {-derivates of } E_{0}^{k}, \ldots ; E_{k-1}^{k}, \\
& =\text { a linear function of the } x \text {-derivates of } E_{1}^{k}, \ldots, E_{k}^{k}, \\
& =0 \text {, by hypothesis. }
\end{aligned}
$$

Since the $x$-derivates of $E_{0}^{k}, E_{1}^{k}, \cdots, E_{k}^{k}$ are given equal to zero, we now know that all the derivates of degree $p-1$ are zero. The same argument applies to the next line of derivates, and so on. Hence the given expression $E$ and all its derivates vanish, so that the system $E$ forms a part, or even the whole, of the one-set system proper to the base-point determined by the two curves. 
28. Proof of Theorem (3).-To determine the number of intersections at the origin of two curves $u, v$ of orders $l, m$, where

$$
\begin{aligned}
& u=a_{0}^{k} x^{k}+a_{1}^{k} x^{k-1} y+a_{2}^{k} x^{k-2} y^{2}+\cdots+a_{0}^{k+1} x^{k+1}+\cdots, \\
& v=b_{0}^{k} x^{k}+b_{1}^{k} x^{k-1} y+b_{2}^{k} x^{k-2} y^{2}+\cdots+b_{0}^{k+1} x^{k+1}+\cdots,
\end{aligned}
$$

let the equation of either curve $w$, arranged by powers of $y$, be

where

$$
w=w_{0}+y w_{1}+y^{2} w_{2}+\cdots=0
$$

$$
\begin{aligned}
& w_{0}=\quad z_{0}^{k} x^{k}+z_{0}^{k+1} x^{k+1}+\cdots, \\
& w_{1}=\quad z_{1}^{k} x^{k-1}+z_{1}^{k+1} x^{k}+z_{1}^{k+2} x^{k+1}+\cdots, \\
& w_{2}=\quad z_{2}^{k} x^{k-2}+z_{2}^{k+1} x^{k-1}+z_{2}^{k+2} x^{k}+z_{2}^{k+3} x^{k+1}+\cdots, \\
& w_{k}=z_{k}^{k}+z_{k}^{k+1} x+\cdots+z_{k}^{2 k-2} x^{k-2}+z_{k}^{2 k-1} x^{k-1}+z_{k}^{2 k} x^{k}+z_{k}^{2 k+1} x^{k+1}+\cdots, \\
& w_{k+1}=z_{k+1}^{k+1}+z_{k+1}^{k+2} x+\cdots+z_{k+1}^{2 k-1} x^{k-2}+z_{k+1}^{2 k} x^{k-1}+z_{k+1}^{2 k+1} x^{k}+z_{k+1}^{2 k+2} x^{k+1}+\cdots,
\end{aligned}
$$
etc.

Then any $u_{h}$ is $w_{h}$ with $a$ written for $z$, and any $v_{h}$ is $w_{h}$ with $b$ written for z. Also $w_{h-1}=D_{y} w_{h}$.

Let $y$ be eliminated from the equations $u=0, v=0$, then the $l m$ values of $x$ corresponding to the points of intersection are to be found by solving the equation

$$
\left|\begin{array}{lllllll}
u_{0} & u_{1} & u_{2} & \cdots & u_{l} & & \\
& u_{0} & u_{1} & & u_{l-1} & u_{l} & \\
& & u_{0} & m \text { rows } & u_{l-2} & u_{l-1} & u_{l} \\
& & \cdots & & & & \\
& & \cdots & & & & \\
v_{0} & v_{1} & v_{2} & & & & \\
& v_{0} & v_{1} & & & v_{m} & \\
& & v_{0} & l \text { rows } & & v_{m-1} & v_{m}
\end{array}\right|=0 .
$$

It is to be determined how many zero roots are present, that is, what power of $x$ is a factor in this determinant. We shall find that the elements of each row can be combined so as to eliminate the lower powers of $x$ only with the help of the equations of the one-set system.

Any expression linear in the $z$ 's with any one index, such as

is the coefficient of $x^{p}$ in

$$
\lambda_{0}^{p} z_{0}^{p}+\lambda_{1}^{p} z_{1}^{p}+\cdots+\lambda_{p}^{p} z_{p}^{p}
$$

$$
\lambda_{0}^{p} w_{0}+\lambda_{1}^{p} x w_{1}+\cdots+\lambda_{p}^{p} x^{p} w_{p}
$$


if however $\lambda_{0}^{p}=0$, this set of terms is, more simply, the coefficient of $x^{p-1}$ in

In general,

$$
\lambda_{1}^{p} w_{1}+\lambda_{2}^{p} x w_{2}+\cdots+\lambda_{p}^{p} x^{p-1} w_{p} .
$$

is the coefficient of $x^{p-s}$ in

$$
\lambda_{s}^{p} z_{s}^{p}+\lambda_{s+1}^{p} z_{s+1}^{p}+\cdots
$$

$$
\lambda_{s}^{p} w_{s}+\lambda_{s}^{p+1} x w_{s+1}+\cdots
$$

Hence the terms given by a more general expression $E_{s}^{p}$, namely,

$$
\lambda_{s}^{p} z_{s}^{p}+\lambda_{s+1}^{p} z_{s+1}^{p}+\cdots+\lambda_{s}^{p-1} z_{s}^{p-1}+\lambda_{s+1}^{p-1} z_{s+1}^{p-1}+\cdots+\cdots,
$$

form the coefficient of $x^{p-s}$ in

$$
\lambda_{s}^{p} w_{s}+\lambda_{s+1}^{p} x w_{s+1}+\lambda_{s+2}^{p} x^{2} w_{s+2}+\cdots+x\left\{\lambda_{s}^{p-1} w_{s}+\lambda_{s+1}^{p-1} x w_{s+1}+\cdots\right\} .
$$

that is, in

$$
+x^{2}\left\{\lambda_{s}^{p-2} w_{s}+\lambda_{s+1}^{p-2} x w_{s+1}+\cdots\right\} \cdots
$$

$$
\left(\lambda_{s}^{p}+x \lambda_{s}^{p-1}+x^{2} \lambda_{s}^{p-2}+\cdots\right) w_{s}+\left(x \lambda_{s+1}^{p}+x^{2} \lambda_{s+1}^{p-1}+\cdots\right) w_{s+1}+\cdots \cdots,
$$

or, say, in $S=X_{s} w_{s}+X_{s+1} w_{s+1}+\cdots \cdots$.

The coefficient of the next lower power of $x$ in this expression $S$ is obtained by taking one step to the eft in every $w$; this replaces $z_{q}^{p}$ by $z_{q}^{p-1}$, that is, by its $x$-derivate. Hence the coefficient of $x^{p-s-1}=D_{x}$. coefficient of $x^{p-s}$, and so on. If then $E_{s}^{p}=0$ is an equation of the one-set system, not only is the coefficient of $x^{p-s}$ in $S$ zero, but also the coefficient of every lower power of $x$. Hence $x^{p-8+1}$ is the lowest power of $x$ that is present in this combination of $w$ 's. If now the columns of the determinant are multiplied by the expressions thus indicated, namely, the $(s+1)$-th by $X_{s}$ (which, it is to be noted, does not contain $x$ as a factor), the $(s+2)$-th by $X_{s+1}$, etc., the $(s+1)$-th column can be replaced by

$$
\begin{aligned}
& X_{s} u_{s}+X_{s+1} u_{s+1}+X_{s+2} u_{s+2}+\cdots \\
& X_{s} u_{s-1}+X_{s+1} u_{s}+X_{s+2} u_{s+1}+\cdots \\
& X_{s} u_{s-2}+X_{s+1} u_{s-1}+X_{s+2} u_{s}+\cdots \\
& X_{s} v_{s}+X_{s+1} v_{s+1}+X_{s+2} v_{s+2}+\cdots \\
& X_{s} v_{s-1}^{\cdot}+X_{s+1} v_{s}+X_{s+2} v_{s+1}+\cdots
\end{aligned}
$$


As regards the $u$-rows and $v$-rows separately, each row is the $y$-derivate of the row immediately above it; hence if the coefficient of $x$ vanishes in the first row, it vanishes in all following rows, that is, the lowest power of $x$ that is present in the first row is a factor in every member of the column. Thus by means of the equation of degree $p$ that heads the $(s+1)$-th column of the particular arrangement of the equations given in $\S 26$ we can make $x^{p-s+1}$ a factor in the $(s+1)$-th column of the determinant, and this is the highest power of $x$ that can be obtained as a factor in this column in this manner. Since $X_{s}$ does not contain $x$ as a factor, no irrelevant powers of $x$ have been introduced in the process. Consequently we obtain, in the first $k$ columns of the determinant, powers of $x$ with exponents $p-(s-1)$, that is, with exponents

$$
p_{0}+1, \quad p_{1}, \quad p_{2}-1, \cdots, \quad p_{k-1}-(k-2) ;
$$

$x$ presents itself therefore as a factor with the exponent

$$
\left(p_{0}+1\right)+p_{1}+\left(p_{2}-1\right)+\cdots+\left(p_{c-1}-\overline{s-2}\right)+\cdots+\left(p_{k-1}-\overline{k-2}\right),
$$

which is the total number of equations in the system. Hence the number of intersections is at least as great as the number of equations in the one-set system.

29. It has still to be shown that no higher power of $x$ is present as a factor. Returning to the original determinant, let the columns be combined so as to replace the $(s+1)$-th by

$$
\begin{gathered}
X_{s} u_{s}+X_{s+1} u_{s+1}+\cdots \\
X_{s} u_{s-1}+X_{s+1} u_{s}+\cdots \\
: \cdot \cdot \cdot \cdot \cdot \cdot \cdot \cdot \\
\cdot \cdot \cdot \cdot \cdot \cdot \cdot \cdot \cdot \\
X_{s} v_{s}+X_{s+1} v_{s+1}+\cdots \\
X_{s} v_{s-1}+X_{s+1} v_{s}+\cdots
\end{gathered}
$$

where the $X$ 's are expressions in $x$, about which nothing is known except that $X_{s}$ does not contain $x$ as a factor. Then if $x^{t+1}$ is a factor in every member of the column, it is a factor in the determinant. In every member of the column the coefficient of $x^{t}$ is zero, as also the coefficient of every lower power of $x$. Now forming the expression $X_{s} w_{s}+X_{s+1} w_{s+1}+\cdots$, and denoting the coefficient of $x^{t}$ in this by $E$, we see that the coefficient of $x^{t-1}$ is $D_{x} E$, and so on ; we see moreover that the coefficient of $x^{t}$ in $X_{s} w_{s-1}+X_{s+1} w_{s}+\cdots$ is $D_{y} E$, and so on. If then $x^{t+1}$ is a factor in the first $k+1 u$-rows, and in the first $k+1$ $v$-rows, it follows that for the two curves $u, v$ the expression $E$, with $k$ successive $y$-derivates, and all their $x$-derivates, are zero. Consequently, by the theorem of $\S 27, E$ is a member of the system proper to the base-point determined by these two curves. This argument does not apply, however, if the expression 
$E$ has no $x$-derivates; that is, if $E$ involves only $z_{k}^{k}, z_{k+1}^{k+1}, z_{k+2}^{k+2}, \ldots$ In this case the combination of $w$ 's obviously begins with $w_{k}$ or a later one; to make even a single $x$ a factor in such a combination, it is necessary that there be values of $\lambda_{1}, \lambda_{2}, \cdots$ satisfying the equations

$$
\begin{array}{r}
z_{k}^{k}+\lambda_{1} z_{k+1}^{k+1}+\lambda_{2} z_{k+2}^{k+2}+\cdots=0 \\
\lambda_{1} z_{k}^{k}+\lambda_{2} z_{k+1}^{k+1}+\cdots=0 \\
\lambda_{2} z_{k}^{k}+\cdots=0
\end{array}
$$

for both $u$ and $v$. In other words, there must be quantities $\lambda_{1}, \lambda_{2}, \ldots$ that satisfy the $m$ equations obtained from these by writing $a$ for $z$, and the $l$ equations obtained by writing $b$ for $z$.

Again, writing $x=0$ in the equations $u=0, v=0$, and dividing by $y^{k}$, we obtain the points other than the origin where these two curves meet the axis of $y$, by means of the two equations

$$
\begin{aligned}
& a_{k}^{k}+a_{k+1}^{k+1} y+a_{k+2}^{k+2} y^{2}+\cdots+a_{l}^{l} y^{l-k}=0 \\
& b_{k}^{k}+b_{k+1}^{k+1} y+b_{k+2}^{k+2} y^{2}+\cdots+b_{m}^{m} y^{m-k}=0
\end{aligned}
$$

The condition that these two equations have a common root is the vanishing of the determinant

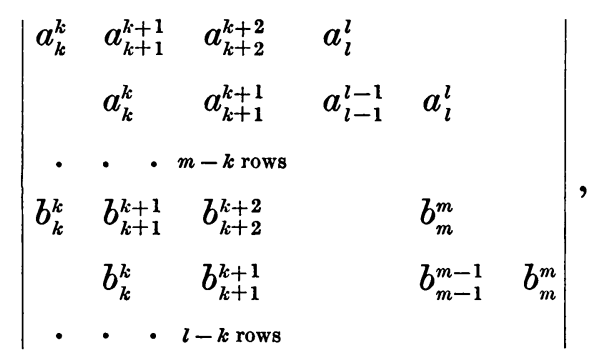

which is precisely the condition that the $m-k$ equations

$$
\begin{aligned}
& a_{k}^{k}+\lambda_{1} a_{k+1}^{k+1}+\lambda_{2} a_{k+2}^{k+2}+\cdots=0 \\
& \lambda_{1} a_{k}^{k}+\lambda_{2} a_{k+1}^{k+1}+\cdots=0 \\
& \text { etc. }
\end{aligned}
$$

and the $l-k$ equations

$$
\begin{array}{r}
b_{k}^{k}+\lambda_{1} b_{k+1}^{k+1}+\lambda_{2} b_{k+2}^{k+2}+\cdots=0 \\
\lambda_{1} b_{k}^{k}+\lambda_{1} b_{k+2}^{k+2}+\cdots=0
\end{array}
$$


be consistent. But these equations (II) are a part of equations (I); if then equations (I) hold, that is, if $x$ is a factor in the combination, equations (II) show that this is due to the fact that the two curves have a point of intersection on the axis of $y$, but distinct from the origin. This depends on a specialized choice of axes, and can be avoided. It is the only way in which a power of $x$ can present itself as a factor without the help of the equations of the one-set system; hence the number of intersections at the origin is that found in $\S 28$, which completes the proof of the

Theorem: the number of intersections of two curves at the origin is equal to the number of equations in the one-set system.

30. In applying the theory to particular curves, one point worthy of notice is that the degree of the prime equation may be higher than the order of either curve by which it is determined. This is natural, inasmuch as the equations relate not only to the given curves $u$, $v$, but to every curve of the system $X u+Y v$. From another point of view, the degree of the prime equation simply tells to what order of small quantities the branches through the origin are specified, and this has no connection with the order of the curve. The equations, regarded as imposing conditions on curves of order $n, n<p$, are subject to mutilation, since all $z$ 's for which the index is greater than $n$ are now zero. But these missing terms must be supplied in any general application of the theory. For instance, the cubics

$$
\begin{aligned}
& y-x^{2}-y^{2}+x^{2} y-x y^{2}+y^{3}=0, \\
& y-x^{2}+x y-x^{3}-x y^{2}=0,
\end{aligned}
$$

have nine-point contact at the origin. The prime equation, of degree 8 , and its $x$-derivates, are

$$
\begin{aligned}
& E^{8}=z_{0}^{8}+z_{1}^{7}+z_{2}^{6}+z_{3}^{5}+z_{1}^{4}-z_{1}^{3}+2 z_{2}^{3}+z_{1}^{2}-2 z_{2}^{2}+z_{1}^{1}=0, \\
& E^{7}=z_{0}^{7}+z_{1}^{6}+z_{2}^{5}+z_{3}^{4}+z_{1}^{3}-z_{1}^{2}+2 z_{2}^{2}+z_{1}^{1}=0, \\
& E^{6}=z_{1}^{5}+z_{0}^{6}+z_{2}^{4}+z_{3}^{3}+z_{1}^{2}-z_{1}^{1}=0, \\
& E^{5}=z_{0}^{5}+z_{1}^{4}+z_{2}^{3}+z_{1}^{1}=0, \\
& E^{4}=z_{0}^{4}+z_{1}^{3}+z_{2}^{2}=0 \\
& E^{3}=z_{0}^{3}+z_{1}^{2}=0 \\
& E^{2}=z_{0}^{2}+z_{1}^{1}=0 \\
& E^{1}=z_{0}^{1}=0 \\
& E^{0}=z_{0}^{0}=0 .
\end{aligned}
$$


The $y$-derivates are linear functions of these; hence the single column of equations gives the complete set. Now as applied to the cubic these equations reduce to

$$
\begin{aligned}
& E^{8}=-z_{1}^{3}+2 z_{2}^{3}+z_{1}^{2}-2 z_{2}^{2}+z_{1}^{1}=0, \\
& E^{7}=z_{1}^{3}-z_{1}^{2}+2 z_{2}^{2}+z_{1}^{1}=0, \\
& E^{6}=z_{3}^{3}+z_{1}^{2}-z_{1}^{1}=0, \\
& E^{5}=z_{2}^{3}+z_{1}^{1}=0 \\
& E^{4}=z_{1}^{3}+z_{2}^{2}=0 \\
& \text { etc., }
\end{aligned}
$$

and these mutilated equations are equivalent to eight only, on account of the relation $E^{8}+E^{7}-E^{5}=0$, which does not hold for the proper equations. Nevertheless the one-set system of equations contains nine members, even when applied to the cubic ; strictly the order of the curve is irrelevant, since the equations relate to the system $X u+Y v$, for which this reduction in number does not take place.

31. A remark in Dr. Macaulay's second paper may properly be noted here. He points out that the equations can be arranged in such an order that stopping at any point, we have the equations of a base point. All that is necessary for this is that no one of the equations shall appear before any of its own derivates. If we break off at any point in the series we thus have a number of sources with all their derivates; that is, the equations proper to a certain $t$-set point, contained in the given one-set point.

\section{Conclusion.}

32. In conclusion, a few remarks of a general character may not be out of place. The fundamental idea of the theory, namely, that the equations can be dealt with as derivates of a comparatively small number, seems to be of real importance. In these pages $I$ have dwelt on certain aspects of the question, hoping to attract other minds to it; for while I believe that the somewhat lengthy presentation here made may possibly be much simplified, yet I confess I cannot see in what direction. The ideas involved are simple and direct, even if their development be somewhat tedious. It seems that more may be done by the direct discussion of the equations, here attempted, than by the ingenious but artificial processes of the original memoirs.

One question as to which there is scope for investigation relates to the geometrical interpretation, not only of the individual equations (which is to some extent answered in $\S 31$ ), but of the diagram by which the whole set is repre- 
sented. It has been shown that the equations arranged as in $\S 10$ present one of the following appearances

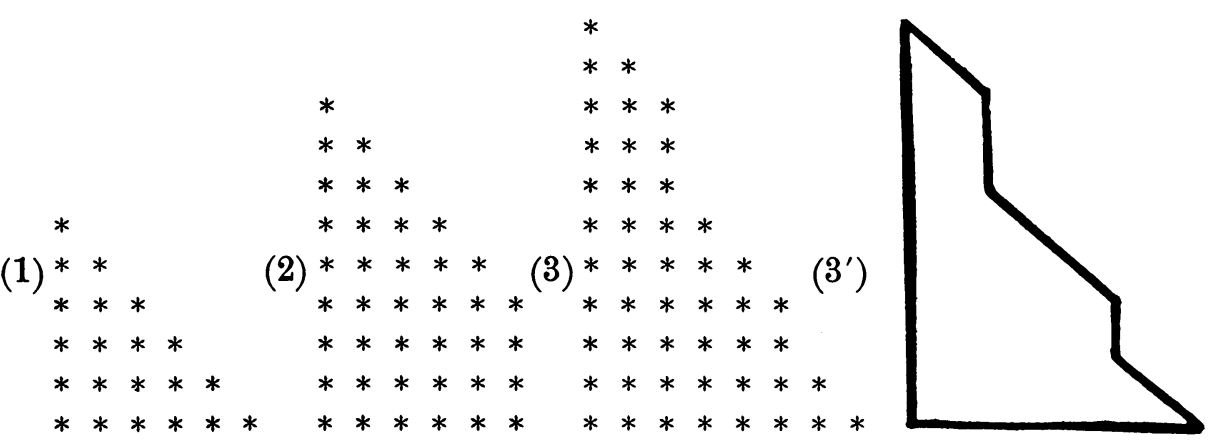

where (3) may have any number of steps, each accounted for as in $\S 10$. Figures (1) and (2) are easily interpreted. If the curves have each a $k$-point, with no contacts, the scheme of equations is of the simple form (1); the prime equation is of degree $2(k-1)$. If one curve has a $k$-point, and the other an $h$-point, where $h>k$, and there is no contact (the simple case when NoETHER's theorem is in question) the diagram is of the form (2). This may alsa be looked upon as representing two curves each with a $k$-point, and with contact of a certain kind. The prime equation is of degree $h+k-2$. This set gives for the number of intersections the sum of $k$ terms

$$
h+k-1, h+k-3, h+k-5, \quad \cdots, \text { that is, } h k . \quad \text { (See } \S 26 .)
$$

As to (3), most probably the vertical boundaries on the right indicate contacts of some of the branches of the two curves, the number of branches involved being shown by the breadth of the section of the figure. This point appears to be well worth investigation; it looks very much as though the form of the diagram might turn out to be a complete indication of the relation of the two curves to one another. Whether the idea will be of any use in the investigation of compound singularities appears doubtful, since the conditions for superlinear branches do not involve the coefficients linearly, and one would hesitate to undertake the discussion of the theory even of quadratic prime and derived equations.

Bryn Mawr College, Pennsyluania. 